\title{
Strategies for the Separation of the Furanic Compounds HMF, DFF, FFCA and FDCA from lonic Liquids
}

\author{
Amir Al Ghatta, James D. E. T. Wilton Ely* and Jason P. Hallett*
}

Department of Chemical Engineering, Imperial College, South Kensington Campus, London SW7 2AZ. Department of Chemistry, Imperial College, Molecular Sciences Research Hub, White City Campus, London W12 OBZ.

e-mail: j.wilton-ely@imperial.ac.uk, j.hallett@imperial.ac.uk

Keywords: biorenewables, ionic liquids, biorefinery, platform chemicals, sustainable chemistry, separations

\begin{abstract}
The catalytic upgrading of sugar derivatives into valuable building blocks represents an extremely important challenge intrinsic to the attempts to establish a green economy. However, the significance of separation and purification are often relegated to a marginal role or overlooked completely despite this aspect being critical for potential scale up. It is well established that the synthesis of 5-hydroxymethylfurfural (HMF) from sugars in ionic liquid media is a valuable, sustainable and high-yielding chemical pathway, but product separation has always remained an unresolved issue. In this contribution, the separation of HMF and three of its derivatives, 2,5diformylfuran (DFF), 5-formyl-2-furancarboxylic acid (FFCA) and 2,5-furandicarboxylic acid (FDCA) from ionic liquids is analyzed. Various ionic liquids are screened in order to obtain an optimal separation process. The extraction of HMF is studied from the hydrophobic methyltrioctylammonium ionic liquids with water, obtaining a favorable partition coefficient for the aqueous phase. In contrast, its derivatives, DFF, FFCA and FDCA, can be easily separated by phase separation. DFF retains its sublimation attributes in the ionic liquid and can be readily separated in quantitative yields in high purity. This behavior is observed in ionic liquids but is not achievable in common organic solvents. FDCA and FFCA are separated by water addition and precipitation. It is found that less water is required for the precipitation of these compounds compared to dimethylsulfoxide (DMSO), which is a frequently employed reaction medium for their generation. The energy balance for regeneration of the ionic liquid after water addition is estimated using the enterprise ionic liquids database ILUAM. This study provides a set of solvent design guidelines for the selective synthesis, isolation and purification of these compounds in ionic liquids, aiding future reaction design.
\end{abstract}

\section{INTRODUCTION}

The depletion of oil reserves and the increasing legislation aimed at limiting $\mathrm{CO}_{2}$ emissions have driven research activities towards the quest for sustainable options. The need for effective utilization of resources and the development of suitable processes to achieve this will shape the future of the chemical industry. Incentives have already been introduced to favor the substitution of chemical products derived from petrochemical sources with renewable feedstocks (such as biomass) and processes that use harmful and toxic chemicals have been banned. ${ }^{1}$

Solvent use in the chemical industry plays a crucial role in defining the sustainability and the environmental impact of the chemical processes involved. ${ }^{2}$ Apart from fuels, solvents represent the greatest quantity of all chemicals used. Around 20 million tons per year are used in the chemical industry, most of which are disposed of by flaring to generate energy since their recovery is economically unattractive due to their low cost. ${ }^{3}$ Besides the damaging environmental impact of such practices, the choice of solvent in a chemical process is a crucial factor to determine the process feasibility of the plant, since many different aspects depend on the solvent, such as reaction compatibility and product separation. ${ }^{4}$ No widely-applicable guidelines are available to guide solvent usage in terms of separation and isolation, so studies have to be carried out on a case by case basis. An attempt to address this issue becomes even more timely now that new classes of solvents are increasingly being studied as potential substitutes for organic solvents. ${ }^{5}$ 
Ionic liquids (ILs) have been proposed as solvents capable of replacing traditional organic options for a large variety of applications. ${ }^{6}$ Their negligible vapor pressure, non-flammability, high thermal stability and, in some cases, low toxicity, make them attractive potential solutions for chemical plants, both in terms of human and environmental safety as well as recyclability. Ionic liquids have proven to be very efficient media for biomass fractionation and chemical transformations based on cellulose, sugars and their derivatives. ${ }^{7,8}$ In this field, the synthesis of 5-hydroxymethylfurfural (HMF) from glucose and fructose has proven to be very efficient in ionic liquids compared to other water-based systems, where low concentrations and biphasic conditions are needed in order to ensure acceptable yields. ${ }^{9-11}$ Very high yields in short reaction times have been achieved in ionic liquids and many catalysts, both homogeneous and heterogeneous, have been developed to increase the rate and selectivity of the reaction in a variety of ionic liquids with different anion and cation combinations. ${ }^{12-16}$ HMF is a valuable platform chemical for the production of many different compounds used in consumer products or as precursors for further reactions. ${ }^{17}$ The scale-up of the production of this platform chemical provides an opportunity for a green process where renewable feedstocks and green processing using ionic liquids are exploited with limited or no use of organic solvents. However, the synthesis of HMF in ionic liquids has never achieved industrial application due both to the high cost of ILs and unresolved separation issues. ${ }^{18}$ The extremely low vapor pressure associated with ILs allows the distillation of volatile products from the medium, but product separation from ILs is compromised for HMF due to the high boiling point and low thermal stability of HMF.

The problem of HMF separation has been addressed in various reports which discuss a variety of different techniques. Thermal distillation under very high vacuum and high temperature $\left(180^{\circ} \mathrm{C}\right)$ has been proposed, despite the risk posed by the thermal instability of $\mathrm{HMF}$, while extraction with $\mathrm{CO}_{2}$ has proven to be successful on a small scale. The feasibility of this latter approach on a larger scale is doubtful due to the low partition coefficient and harsh conditions used. ${ }^{19-21}$ Extraction with organic solvents, such as ethyl acetate, is regularly used in many reports but the partition coefficient is unfavorable, necessitating excessive washing. ${ }^{22}$ Moreover, the use of organic solvents during product recovery undermines some of the benefits of using ILs, since this introduces flammable compounds to the system and presents other disadvantages associated with organic solvents, such as volatility and toxicity. These are often the same issues the use of an ionic liquid was meant to avoid. In this context, the in situ conversion of HMF to a chemical compound with less challenging separation properties represents a potential solution. Accordingly, three very useful products have been identified that can be produced through aerobic oxidation starting from HMF (Figure 1) and, importantly, can also be easily separated from ILs; 2,5-diformylfuran (DFF), 5-formylfuran2-carboxylic acid (FFCA) and 2,5 furandicarboxylic acid (FDCA). While DFF and FFCA have potential in the pharmaceutical and consumer product industry, ${ }^{23,24}$ FDCA is the key monomer for the production of polyethylene furanoate (PEF), a bioplastic suitable for use as a food and beverage packaging in place of polyethylene terephthalate $(\mathrm{PET})$.

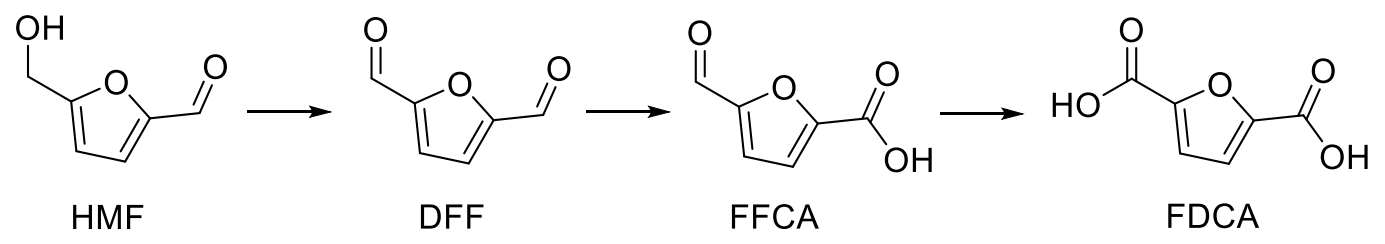

Figure 1. Reaction pathway to obtain high value-added chemical intermediates from ionic liquids.

The market demand for FDCA is high, but its production on an industrial scale is limited by the low isolated yield of HMF from sugars. ${ }^{25}$ Many systems have been reported for the selective oxidation of HMF to these chemicals based on both noble and non-noble metal catalysts in water or organic solvents. Solvent effects have proved to be a key factor in the selectivity of the reaction with the water content playing an important role in the system. Organic solvents have proved to be more selective towards DFF, ${ }^{26-31}$ while water is the most commonly used solvent for the synthesis of the carboxylic acids FFCA and FDCA. ${ }^{25,32-34}$ 
In this contribution, the separation of the four molecules shown in Figure 1 from various ionic liquids will be reported. The aim is to identify the most suitable ionic liquid(s) for each compound in terms of product separation and thus to provide guidelines for a solvent design strategy to enable the successful synthesis and scaled up production of these molecules. The separation of these compounds will also need to be compatible with the catalytic system used. In the case of homogeneous catalysis, precipitation of the final product from the reaction mixture is preferred in order to avoid expensive thermal separation and favor catalyst recycling. In contrast, under heterogeneous catalytic conditions, the final product should be soluble to avoid catalyst deactivation. Separation in this case has to be performed using heating or by addition of an antisolvent after catalyst removal. Recently, important progress has been made in the oxidation of HMF and sugars to FDCA in [bmim]Cl but the results described only report HPLC yields, ${ }^{35-37}$ hence separation and purification will play a determinant role in defining the feasibility of scale-up using ionic liquid technology for this application.

Recently, Srinivasan and co-workers analyzed the separation of glucose, HMF, DFF and FDCA from dimethylsulfoxide (DMSO) by antisolvent addition and precipitation. ${ }^{38}$ In their work, glucose was separated by addition of organic solvents, DFF and FDCA by water addition and HMF by addition of water followed by separation using solvent extraction. Many papers report DMSO as efficient for both sugar dehydration and HMF oxidation, ${ }^{39-}$

${ }^{41}$ however, the applicability of this solvent on a large scale is hindered by the high boiling point of the solvent (189 ${ }^{\circ} \mathrm{C}$ ), its decomposition to toxic sulfur compounds and the need for low substrate loadings in many cases. Moreover, a process based on DMSO would still be dependent on organic solvents and separation though antisolvent addition, which can result in high energy requirements for solvent regeneration. However, an energy balance analysis would be needed to evaluate the techno-economic impact of such a process.

In order to minimize the use of organic solvents, the extraction of HMF, DFF, FFCA and FDCA from ionic liquids was investigated in this work in order to establish the most suitable ionic liquid for each compound to allow further processing. Where the lack of volatility of the ILs caused difficulties in product separation, this property was exploited, along with the polarity of the ILs, to achieve an optimum separation of the components from the mixtures. Imidazolium ionic liquids were investigated for the separation of these compounds for the low viscosity. This was preferred since the catalytic aerobic oxidation route which lead to DFF, FFCA and FDCA requires solvents with low viscosity due to mass transfer limitations. In the context of this work, the aim was to demonstrate that the one-pot synthesis of these compounds in the ionic liquid can provide a more favorable environment for their subsequent separation. Moreover, separation through sublimation and precipitation are strongly affected by viscosity since it can limit the formation of crystals and hence reduce the rate of separation. The investigation of different anions allowed the effect of hydrogen-bond acceptor ability on the separation to be assessed. The study of HMF was carried out with ammonium ionic liquids in order to gauge the hydrophobicity effect on the extraction with water. The final extraction of HMF in water provided a suitable environment for subsequent oxidation to FDCA which can be easily separated from water by lowering the $\mathrm{pH}$.

Most literature reports on the synthesis of DFF use organic solvents, ${ }^{31,42}$ raising doubts over the feasibility of potential scale up due to product separation issues and environmental sustainability. Based on our own observations and previous work, ${ }^{43}$ DFF cannot be effectively sublimed from organic solvents (either upon heating or under vacuum) but this property is retained in ionic liquids. Hence, the synthesis of DFF in ionic liquids allows separation by sublimation and this represents a solution to large-scale product separation as well as solvent recycling without the need for expensive thermal treatments. Moreover, sublimation usually delivers a high purity product, avoiding the need for further purification.

The separation of FDCA and FFCA was studied by calculating their solubility in different ionic liquid - water mixtures. Only recently has FDCA been successfully prepared from HMF in [bmim]Cl using a Fe/Zr catalyst, making this system compatible with the formation of HMF from glucose. ${ }^{37}$ While this system has the potential to deliver a one-pot synthesis of this plastic monomer from sugars, separation still remains a challenge. In this contribution, the separation of FDCA from ionic liquids was studied through water addition, exploiting the low solubility of FDCA in water. However, the addition of water to a system based on ionic liquids requires regeneration of the IL solvent for further catalytic cycles. Using the ILUAM database package for ionic liquids, ${ }^{44}$ a multi-effect evaporation process was simulated to estimate the energy requirement for regeneration of the ionic liquid. Compared to the values 
reported for DMSO, ${ }^{38}$ this reveals that the addition of a smaller quantity of water is required when an ionic liquid is used (vide infra), decreasing the energy and water consumption for potential scale up.

For HMF, hydrophobic ionic liquids were studied based on methyltrioctylammonium salts paired with water extraction of HMF. Ammonium salts have been reported as good solvents for the dehydration of glucose to HMF in high yield, ${ }^{45}$ showing minimal leaching into the water phase. ${ }^{46}$ The combination of ammonium salts with water could form the basis of a system free of organic solvents for HMF extraction and subsequent oxidation to FDCA, since water has (thus far) proved to be the most efficient medium for the catalytic oxidation of aldehydes and alcohols to carboxylic acids. ${ }^{25}$ Using hydrophobic ionic liquids, the affinity of HMF towards the water phase can be favored, providing the basis for an improvement in the partition coefficient for the extraction of HMF. Many catalysts have been studied for this oxidation under base-free conditions, making ready implementation of a two-step reaction possible. Previously, different organic solvents were studied to extract HMF from the ionic liquid phase with the chloride-based ionic liquids. An extensive study was reported by Zhou and co-workers ${ }^{47}$ in which different organic solvents were tested for the extraction of HMF from [bmim] Cl, showing that glycol dimethyl ether and acetyl acetone performed best with a partition coefficient close to 3.33 towards the ionic liquid phase over THF, dibutyl ether, methyl tertiary-butyl ether (MTBE), methyl isobutyl ketone (MIBK), cyclohexanone, ethyl acetate and $n$ butanol. Another report shows that by exchanging the chloride anion of the ionic liquid with triflate, the partition coefficient can be increased by an order of magnitude. ${ }^{15}$ We show that a system based on the use of a hydrophobic ionic liquid and water also allows the partition coefficient to be improved remarkably.

\section{EXPERIMENTAL SECTION}

Materials. All material for ionic liquid synthesis and cold finger glassware were purchased from Sigma-Aldrich, as were the methyltrioctylammonium salts with chloride ( $\geq 97 \%)$, bromide $(\geq 97 \%)$ and bistriflimide $(\geq 99 \%)$ anions. Methylimidazole (99\%) was distilled under reduced pressure over $\mathrm{CaH}_{2}$. Chlorobutane $(99.5 \%)$ was distilled at atmospheric pressure over $\mathrm{P}_{2} \mathrm{O}_{5}$. HMF, DFF, FFCA and FDCA were obtained from Fluorochem. Procedures employed for the synthesis of the ionic liquids and for HMF purification are reported in the Supporting Information.

DFF sublimation. The sublimation of DFF was performed using a cold finger. DFF $(40 \mathrm{mg})$ was placed in a $1 \mathrm{~mL}$ vial and mixed with $400 \mathrm{mg}$ of the IL to be tested. The vial was placed inside a cold finger and the substrate was solubilized at the operating temperature for 10 min before a vacuum was applied for the desired time. The DFF was collected on the tip of the condenser, which was cooled to $7^{\circ} \mathrm{C}$. Images of the experimental setup are provided in the Supporting Information (Section S4). All experiments were performed two or more times.

Precipitation of FFCA and FDCA from ionic liquids by water addition. The solubility of FDCA and FFCA was determined at different IL-water mixtures. The saturation point of the mixture was determined by gradual addition of the substrate in a certain amount of solvent $(\sim 300 \mathrm{mg})$ followed by stirring overnight. Samples were centrifuged $(14,800 \mathrm{rpm})$ and the liquid phase was analyzed by HPLC with a UV-detector operating at $254 \mathrm{~nm}$ calibrated with an external standard. Experiments were repeated at least three times.

The energy required for dehydration was calculated using Aspen Plus 8.8v. The ILUAM package was used to simulate multistage effect evaporators. The number of stages and vacuum stages were optimized to obtain the desired water concentration.

HMF extraction from a hydrophobic ionic liquid. The partition coefficient of HMF between the IL and water was calculated by varying the substrate loading, temperature and phase ratio. Experiments were performed in triplicate. A stock solution of the methyltrioctylammonium salts with HMF at the desired concentration $\left(\boldsymbol{C}_{H M F 0}^{I L}\right)$ was prepared on a large scale by dissolving the two compounds in dichloromethane. Aliquat solutions were removed and placed in $1.5 \mathrm{~mL}$ centrifuge vials, from which the organic solvent was removed under vacuum for $12 \mathrm{~h}$. A certain amount of water was then added to each vial in order to obtain the desired phase ratio $(R)$ followed by stirring for $12 \mathrm{~h}$ to 
ensure equilibrium had been reached. The water phase concentration $\left(\boldsymbol{C}_{H M F}^{w}\right)$ of HMF was analyzed by HPLC with external standard calibration and the partition coefficient $(\mathrm{K})$ calculated according to the following equation:

$$
\text { Eq. } 1 \quad \frac{1}{K}=1-R \frac{C_{H M F}^{w}}{C_{H M F 0}^{I L}}
$$

The calculation of the number of washes required to achieve a desired recovery $(\mathrm{y})$ was determined by the following equation:

$$
\text { Eq. } 2 \quad N=\frac{\ln (1-y)}{\ln \left(1-\frac{R}{K+R}\right)}
$$

Analytical method. Quantification of HMF, FFCA and FDCA was performed using a Shimadzu Prominence preparative HPLC (Shimadzu, Kyoto, Japan) with refractive index (RI) and ultraviolet (UV) detectors. This instrument was equipped with an Aminex HPX-87H column using $0.005 \mathrm{M} \mathrm{H}_{2} \mathrm{SO}_{4}$ as the mobile phase at $55^{\circ} \mathrm{C}$ and a flow rate of $0.6 \mathrm{~mL} / \mathrm{min}$.

Calculation of Solubility of Hydrophobic Ionic Liquid in Water. The solubility of the ionic liquid of water was determined by mixing the two phases overnight at room temperature $\left(20^{\circ} \mathrm{C}\right)$. The water phase was analyzed by ion chromatography Dionex ICS-5000+ DC using a method reported in the literature. ${ }^{48}$

\section{RESULTS AND DISCUSSION}

Sublimation of DFF from ionic liquids. The separation of DFF from imidazolium ionic liquids was investigated using the anions chloride and bistriflimide $\left(\left[\mathrm{NTf}_{2}\right]^{-}\right)$, in order to vary hydrogen bond accepting ability. It was observed that DFF starts to sublime slowly under solvent free conditions at $60^{\circ} \mathrm{C}$ without the need for vacuum. However, in order to make the system more efficient, a higher temperature and vacuum were used. At $100{ }^{\circ} \mathrm{C}$ for 12 hours, most of the DFF was recovered at $10 \%$ loading from both ILs (Table 1), showing no influence of the ionic liquid on the sublimation property of DFF.

Table 1. DFF recovered by sublimation at $100{ }^{\circ} \mathrm{C}$ under reduced pressure, $10 \%$ DFF loading, $12 \mathrm{~h}$.

\begin{tabular}{lcc} 
ionic liquid & DFF recovered from commercial sample & DFF recovered from second sublimation \\
\hline$[\mathrm{bmim}] \mathrm{Cl}$ & $93.1 \pm 0.1 \%$ & $96.7 \pm 0.4 \%$ \\
{$[\mathrm{bmim}]\left[\mathrm{NTf}_{2}\right]$} & $95.2 \pm 0.3 \%$ & $97.7 \pm 0.2 \%$ \\
\hline
\end{tabular}

A second sublimation was performed on the same recovered material, achieving a higher recovery, suggesting that DFF was obtained in greater purity than initially present in the commercial sample. This is further confirmed by the fact that the brown as-purchased DFF becomes a white powder on sublimation under our conditions (see Section S4 in Supporting Information for images).

In [bmim] [NTf 2 , DFF showed low solubility at room temperature $(10.2 \pm 0.6 \mathrm{mg} / \mathrm{g})$ so heating at $40{ }^{\circ} \mathrm{C}$ was required to achieve full dissolution of DFF at $10 \%$ loading, indicating that precipitation could be used as another useful separation method since [bmim] $\left[\mathrm{NTf}_{2}\right]$ is a room temperature ionic liquid. While this approach could be useful on a small scale, the high viscosity of the IL and the repeated washing of the product would lead to excessive waste generation and lower yield on a larger scale. For this reason, the precipitation option was not considered further in this study. An investigation conducted in [bmim] [NTf 2 for 6 hours showed that a minimum temperature of $60^{\circ} \mathrm{C}$ is required to fully recover the dissolved DFF by sublimation (Figure 2). In contrast, at $40{ }^{\circ} \mathrm{C}$, DFF is fully dissolved with only $7 \%$ recoverable by sublimation. 


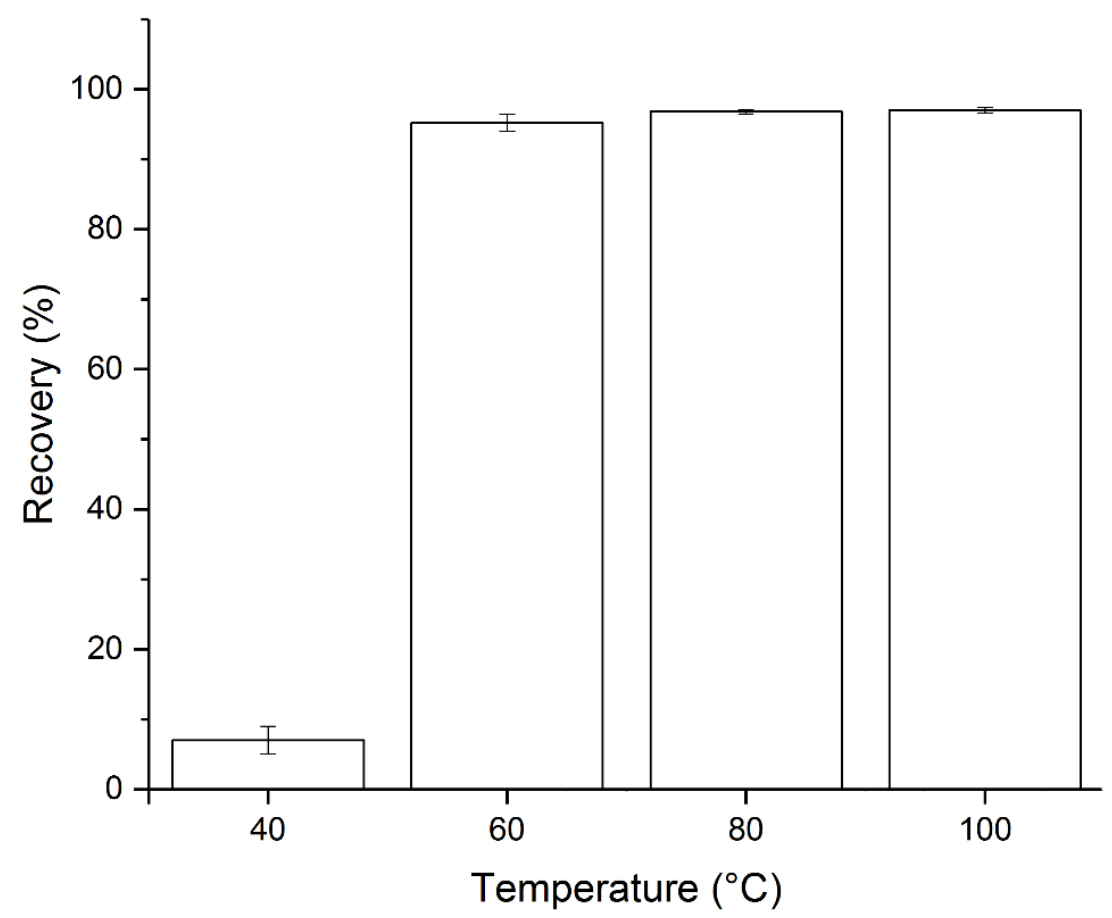

Figure 2. Recovery of DFF in $3 \mathrm{~h}$ at different temperatures from [bmim] $\left[\mathrm{NTf}_{2}\right]$ under vacuum (20 mbar).

The ability of DFF to sublime from the ionic liquid makes this system a viable option to valorize HMF through selective alcohol oxidation. This suggests that the unhelpfully high affinity of HMF for ionic liquids can be circumnavigated by converting the hydroxyl group into an aldehyde and thus minimizing the interaction of the solute with the ions. Wang and co-workers have shown that the major interaction between HMF molecules and the ions in the IL is the hydrogen bonding between the anion and the HMF hydroxyl group, with other interactions being negligible. ${ }^{49}$ The weaker interactions of the aldehyde groups in DFF with the ions in the IL favor its separation by conserving the sublimation properties of DFF. The possibility of exploiting this separation technique makes the scale-up of this reaction very attractive.

Separation of FDCA and FFCA. The relative solubility of FDCA and FFCA in ionic liquids provides a parameter with which to define the potential for separation of these compounds by precipitation. By exploiting the hydrophilicity of the ILs and the known insolubility of these compounds in water, 50 the solubility of these compounds at room temperature was calculated for different water contents. Since halide-based ionic liquids are not room temperature ionic liquids, water contents above $20 \%$ were selected as the minimum amount necessary to achieve a liquid state with sufficiently low viscosity to allow separation at room temperature. The solubility of FDCA in [bmim]Cl at high temperature is reported to be $49 \%$. In this contribution, the solubility of FDCA in both [bmim] Cl and [bmim] $\mathrm{Br}$ were compared for the separation, however, so far, only [bmim] $\mathrm{Cl}$ has been reported as a solvent for the synthesis of FDCA. ${ }^{37}$ The results reported in Figure 3 show that FFCA exhibits higher solubility compared to FDCA in the ILs tested. The higher solubility of FDCA at low water content suggests that the main interactions involve the hydrogen bonding between the carboxylic acid groups and the anions. These interactions are suppressed by an increase in water addition since hydrogen bonding between chloride and the water molecules is stronger than with the carboxylic acid units.

In light of our studies (vide supra) on DFF, the high solubility of FFCA was unexpected considering the lower affinity of the aldehyde group for anions, as demonstrated by Wang and co-workers. ${ }^{49}$ The interaction of water with the aldehyde moiety plays a key role in the behavior of FFCA, as a geminal diol could be formed, which would dramatically increase the solubility of the aldehyde. ${ }^{51}$ At high water content, the interactions that dominate 
are between the water and the aldehyde, however the acidity of the carboxylic acid group in FFCA can hinder the formation of the geminal diol, leading to a decrease in its solubility. At low water content, the molecule can exhibit a combined interaction with both water and chloride anions. This allows water to interact with the aldehyde while hydrogen bonding between the chlorides and the carboxylic acid increases the pKa of the molecule, favoring the formation of diols. Regarding the role of the anion, FDCA and FFCA proved to be less soluble in the bromide-based ionic liquid, probably due to the lower hydrogen-bonding ability of the bromide and the carboxylic group.
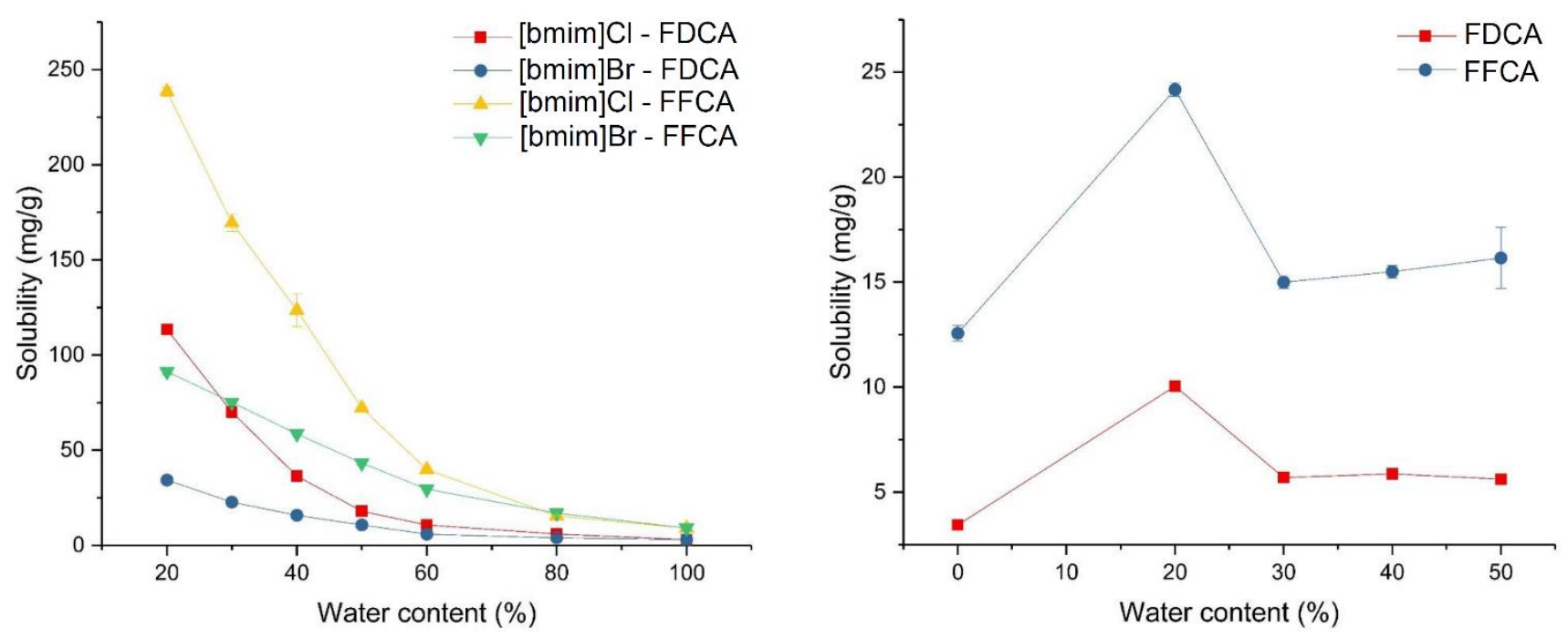

Figure 3. Solubility of FDCA and FFCA in ionic liquid - water mixtures of [bmim] Cl and [bmim]Br (left) and [bmim][OTf] (right) at room temperature; solubility expressed in terms of $\mathrm{mg}$ of compound per gram of solvent.

In order to analyze the role of water on the solubility of FDCA and FFCA in greater depth, a study was carried out with the hydrophilic, non-coordinating ionic liquid [bmim][OTf] over a range of water content. The results reported in Figure 3 (on the right) show a different trend to that exhibited for [bmim]Cl. In pure [bmim][OTf], FDCA shows very low solubility $(<4 \mathrm{mg} / \mathrm{g}$ ), while FFCA demonstrates higher solubility, suggesting that the aldehyde group has a superior affinity for this non-hydrogen acceptor environment. The maximum solubility observed at $20 \%$ water content indicates a synergistic effect between the ionic liquid and water in the dissolution of these compounds. Since this maximum is observed for both compounds, the interactions may specifically involve the carboxylic acid group. Increasing the water content leads to a plateau where the solubility approaches that in pure water. Higher FFCA solubility at higher water content suggests an interaction of the water with the aldehyde group to form geminal diols, which has already been demonstrated in other reports. ${ }^{51,52}$ While outside the scope of the present work, further insight could be obtained using thermodynamic modeling ${ }^{53,54}$ to explain the interaction of the ions and water with FFCA and FDCA.

The data gathered show that both FDCA and FFCA can be separated from [bmim] Cl, [bmim]Br and [bmim][OTf] by addition of water. The results reported by Srinivasan et al in 2017 demonstrate that the solubility of FDCA in DMSO at $20 \%$ water content is around $30 \%{ }^{38}$ Figure 3 (left) shows that this value decreases to $12 \%$ for [bmim] Cl and only $3.4 \%$ in [bmim] Br with the same water content. In this context, ILs represent a suitable potential solution for the reduction of water consumption ahead of a possible scale-up.

\section{Energy analysis of drying [bmim] Cl and [bmim]Br}

The addition of an antisolvent, such as water, can impact drastically on the energy required for solvent regeneration in a process. The energy required to fully dry the IL salts can be high due to the increase in the boiling point of salt solutions and the high heat of vaporization of water $(2.26 \mathrm{MJ} / \mathrm{Kg})$. Multieffect evaporation represents an efficient 
process to reduce the energy requirements by exploiting the heat of vaporization at high pressure to satisfy the energy requirements of the stages operating at lower pressures. In this investigation, we chose four stages at 0.8 , $0.5,0.2$ and 0.01 bar to dehydrate the ionic liquid until $1 \%$ water remained. $20 \mathrm{mg}$ of product (FDCA or FFCA) per gram of the IL-water mixture was chosen as the threshold level of the residual product in the solvent after precipitation. The corresponding solubility values are shown in Figure 3 and data on drying the ILs are reported in Table 2.

Table 2. Energy requirement for the drying of ILs until $1 \%$ water content in 4-stage evaporators with a final pressure of 0.01 bar. Calculations were performed using Aspen Plus $8.8 \mathrm{~V}$ with the ILUAM integrated package.

\begin{tabular}{ccc} 
lonic liquid/product & Initial water concentration & Energy Requirement $(\mathrm{KJ} / \mathrm{Kg})$ \\
\hline$[\mathrm{bmim}] \mathrm{Cl} / \mathrm{FDCA}$ & $52.0 \%$ & 409 \\
{$[\mathrm{bmim}] \mathrm{Br} / \mathrm{FDCA}$} & $37.5 \%$ & 368 \\
{$[\mathrm{bmim}] \mathrm{Cl} / \mathrm{FFCA}$} & $83.5 \%$ & 492 \\
{$[\mathrm{bmim}] \mathrm{Br} / \mathrm{FFCA}$} & $79.0 \%$ & 489 \\
\hline
\end{tabular}

According to Table 2, removal of the quantity of water required to fully precipitate FFCA from [bmim]Cl or [bmim] $\mathrm{Br}$ would lead to high energy requirements, making this approach unsuitable for scale up purposes. For FFCA, [bmim][OTf] is a better solvent choice for valorization due to the low solubility of FFCA in this medium. In contrast, the use of [bmim] $\mathrm{Cl}$ or [bmim] $\mathrm{Br}$ is more favorable for FDCA as less energy is required due to the lower solubility of this product in the IL medium.

A detailed energy analysis for a FDCA plant using ionic liquids would need to be considered in order to optimize the process and minimize operating costs.

\section{Extraction of HMF from trioctylmethylammonium salts using water}

Initially, the effect of various temperatures between 20 and $80^{\circ} \mathrm{C}$ on the partition coefficient for HMF between the hydrophobic salt and water was studied for trioctylmethylammonium chloride, $\left[\mathrm{N}(\mathrm{Me}) \mathrm{Oct}{ }_{3}\right] \mathrm{Cl}$. The data interpolation in this temperature range (Figure S24) is in good agreement with the Arrhenius equation. The partition coefficient towards the water phase is favored by increasing the temperature. This leads to a decrease in the hydrogen bonding interactions between HMF and the IL in favor of secondary interactions between the HMF molecules and the water present.

The feasibility of using water to extract HMF from the IL was evaluated to provide information on the number of washes required to achieve $90 \%$ recovery and a value for the final HMF concentration in the water phase. This reveals that, in order to further valorize HMF into FDCA through aerobic oxidation, a maximum concentration of 0.1 $\mathrm{M}$ in the water phase is advisable since, at higher values, FDCA precipitation would be excessive and lead to catalyst deactivation. In such a scenario a base needs to be added. ${ }^{33}$ However, the final concentration should not be allowed to fall significantly, as lower values would result in substantial water consumption on scale up. The initial loading of HMF and the amount of water used in each stage are crucial factors and these aspects are summarized in Figure 4. Using the ionic liquid $\left[\mathrm{N}(\mathrm{Me}) \mathrm{Oct}_{3}\right] \mathrm{Cl}$, a minimum of $40 \% \mathrm{HMF}$ loading is necessary to achieve a concentration close to $0.1 \mathrm{M}$ and an excessive amount of washing is required. As can be seen in Figure 4 (right) at an $\mathrm{HMF}$ loading of $10 \%$ in the IL, 13 washes are required to extract $90 \%$ of the $\mathrm{HMF}$, leading to a concentration in the aqueous phase of $0.03 \mathrm{M}$. In order to achieve the desired concentration of HMF in the IL, high sugar loadings are needed. A reduction in the amount of washing can be accomplished by increasing the water/IL ratio, but this would reduce the HMF concentration in the water phase. Improved yields are indeed often reported under dilute conditions for the production of HMF from cellulose in ILs, for example. ${ }^{55}$ While this is acceptable on a lab scale, more concentrated systems $\mathrm{s}^{56-58}$ are needed for realistic scale-up. 

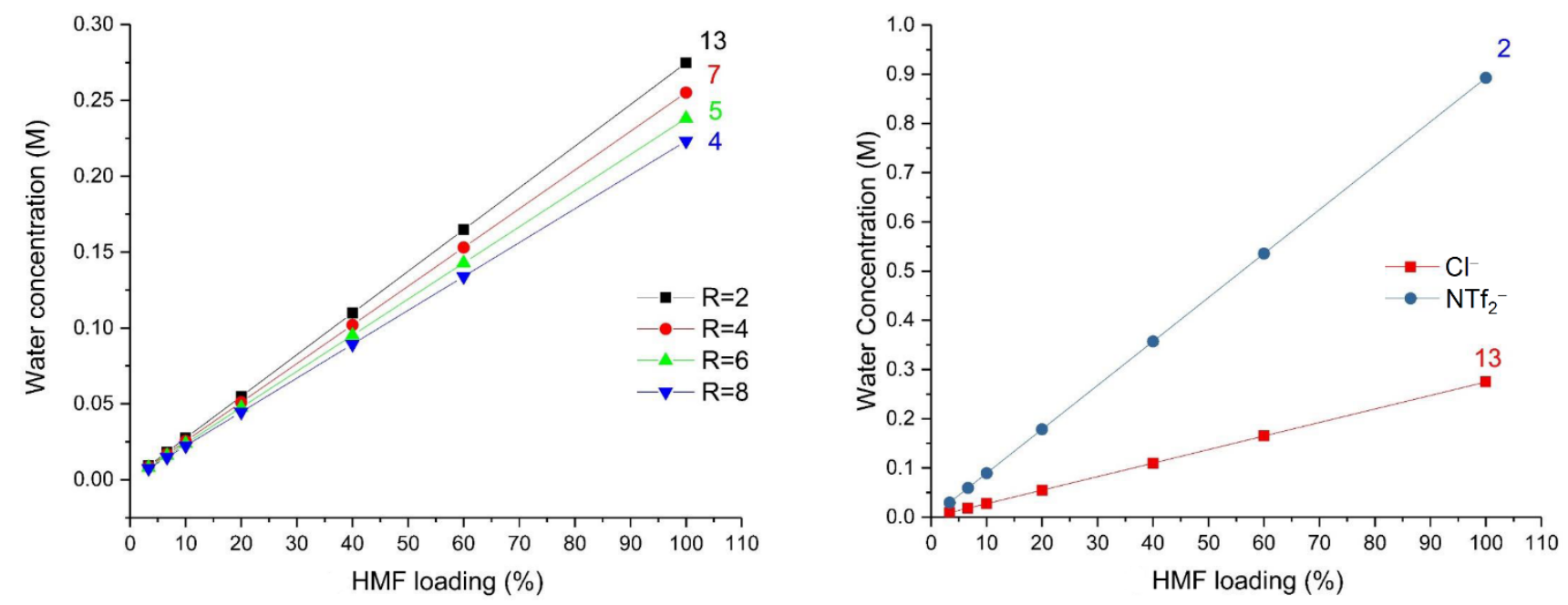

Figure 4. On the left, water concentration to achieve $90 \% \mathrm{HMF}$ recovery from $\left[\mathrm{N}(\mathrm{Me}) \mathrm{Oct}_{3}\right] \mathrm{Cl}$ at different phase ratios (R) and different $\mathrm{HMF}$ loadings at $20^{\circ} \mathrm{C}$. On the right, water concentration needed to achieve $90 \% \mathrm{HMF}$ recovery from $\left[\mathrm{N}(\mathrm{Me}) \mathrm{Oct}_{3}\right] \mathrm{Cl}$ and $\left[\mathrm{N}(\mathrm{Me}) \mathrm{Oct}_{3}\right]\left[\mathrm{NTf}_{2}\right]$ ionic liquids at $\mathrm{R}=2$. Numbers next to the data points represent the number of washes required.

Since the high affinity observed between HMF and ionic liquids is due to the hydrogen bonding with the anion, the non-coordinating ammonium ionic liquid with the bistriflimide ion was tested and it was found that the number of washes was drastically reduced and a higher concentration of HMF in the water phase can be achieved. At $10 \%$ loading, an HMF concentration of more than $0.1 \mathrm{M}$ can be achieved (compared to $0.03 \mathrm{M}$ using the chloridebased IL) with only two washes.

In order to further study the effect of the hydrogen bonding interactions and to improve the partition coefficient, the impact of different anions and cations was studied and the associated partition coefficients were measured (Figure 5).
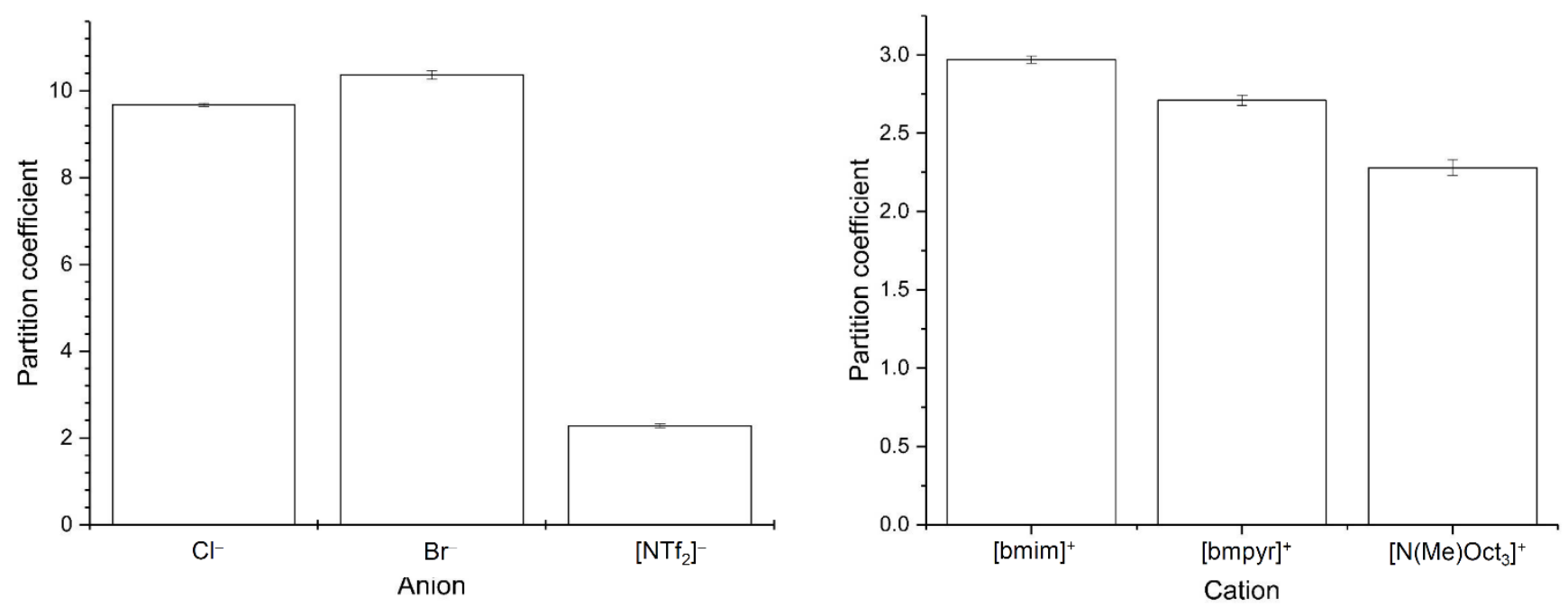

Figure 5. Effect of different anions on the partition coefficient for the $\left[\mathrm{N}(\mathrm{Me}) \mathrm{Oct}_{3}\right]^{+}$salts (left) and the effect of the cation for $\mathrm{NTf}_{2}-$ salts (right).

lonic liquids with anions possessing good hydrogen bonding acceptor ability ( $\mathrm{Br}$ - and $\left.\mathrm{Cl}^{-}\right)$proved to have a high partition coefficient towards the ionic liquid phase, while the non-coordinating $\left[\mathrm{NTf}_{2}\right]^{-}$favors partition towards 
the water phase, making it ideal for the purposes of extraction. The cations were also found to play a significant role. By taking the Kamlet-Taft parameters as a reference point, the high hydrogen donating ability ( $\alpha)$ of [bmim] $]^{+}$ resulted in a greater affinity of HMF for the ionic liquid, compromising the extraction efficiency, while the $\left[\mathrm{N}(\mathrm{Me}) \mathrm{Oct}_{3}\right]^{+}$salts with lower hydrogen donating ability gave the most favorable values. According to these results, the cation has an influence on the partition coefficients, suggesting that interactions with the hydroxyl group or aldehyde unit are present. In order to shed light on these interactions, infrared spectroscopic analysis was performed on samples of HMF in various ionic liquids in order to analyze the shifts of the hydroxyl $(\mathrm{O}-\mathrm{H})$ and carbonyl $(\mathrm{C}=\mathrm{O})$ bonds due to their interactions with the anions and cations present. A blue shift indicates a peak which shifts towards a higher wavenumber value compared to the solvent-free interactions. This indicates the breaking of hydrogen bonds, while the opposite behavior is indicated by a red shift in the spectroscopic feature. As observed by Wang and co-workers, ${ }^{49}$ increasing the mole fraction of ionic liquid causes the signal of the hydroxyl group to become broader and less intense. In order to observe the effect of the ionic liquid on the IR spectroscopic features associated with HMF, up to $30 \%$ (by mole fraction) of the ionic liquids could be added to the HMF. Above this value, the hydroxyl feature was no longer invisible. Figure 6 reveals that the anion plays a major role in the interactions with both the hydroxyl and carbonyl groups of HMF. In the hydroxyl region of the infrared spectra, all the ionic liquids exhibit red shifts from the solvent-free HMF signal at $3366 \mathrm{~cm}^{-1}$ (the IR spectrum of pure HMF is reported in Figure S25 of the Supporting Information) in the order $\mathrm{Cl}^{-}>\mathrm{Br}^{-}>\left[\mathrm{NTf}_{2}\right]^{-}$. The bistriflimide anion showed a lower hydrogen-bonding ability (Figure 6, left) with a slight blue shift at $3391 \mathrm{~cm}^{-1}$ in $\left[\mathrm{N}(\mathrm{Me}) \mathrm{Oct}_{3}\right]\left[\mathrm{NTf}_{2}\right]$, while the chloride and bromide analogues exhibit red shifts of $3262 \mathrm{~cm}^{-1}$ and $3337 \mathrm{~cm}^{-1}$, respectively, confirming the higher hydrogen-bonding ability of the halide anions for coordination to the hydroxyl group. This enhanced interaction of the chloride anion with the hydroxyl group provides an explanation for the greater solubility of FDCA and FFCA indicated in Figure 3. However, the results in Figure 5 show a different trend compared to the hydrogen-bonding ability of the anion, since a higher partition coefficient is observed for the bromide anion, suggesting that other interactions are involved. The spectra in Figure 6 (right) show that the carbonyl region undergoes a slight red shift from the original value of $1683 \mathrm{~cm}^{-1}$, with the greatest belonging to the bromide IL $\left(1667 \mathrm{~cm}^{-1}\right)$ and the smallest to the bistriflimide solvent $\left(1678 \mathrm{~cm}^{-1}\right)$, while the presence of the chloride anion leads to an intermediate shift (1674 $\mathrm{cm}^{-1}$ ). The effect of the cation on both functional groups of HMF was negligible (Figure S26 in the Supporting Information) indicating little relevant contribution by the cation to hydrogen-bonding. The polarizability of the ionic liquid is an alternative indicator of the more favorable partition coefficient for the ammonium salt and lesser interaction with the furan ring (Figure S26 in the Supporting Information). In particular, it was observed that the $\mathrm{HMF}$ and the ammonium salt do not exhibit full miscibility as found for the [bmim $]^{+}$and [bmpyr $]^{+}$solvents, suggesting that secondary interactions arise between the cation and the furan ring of HMF.
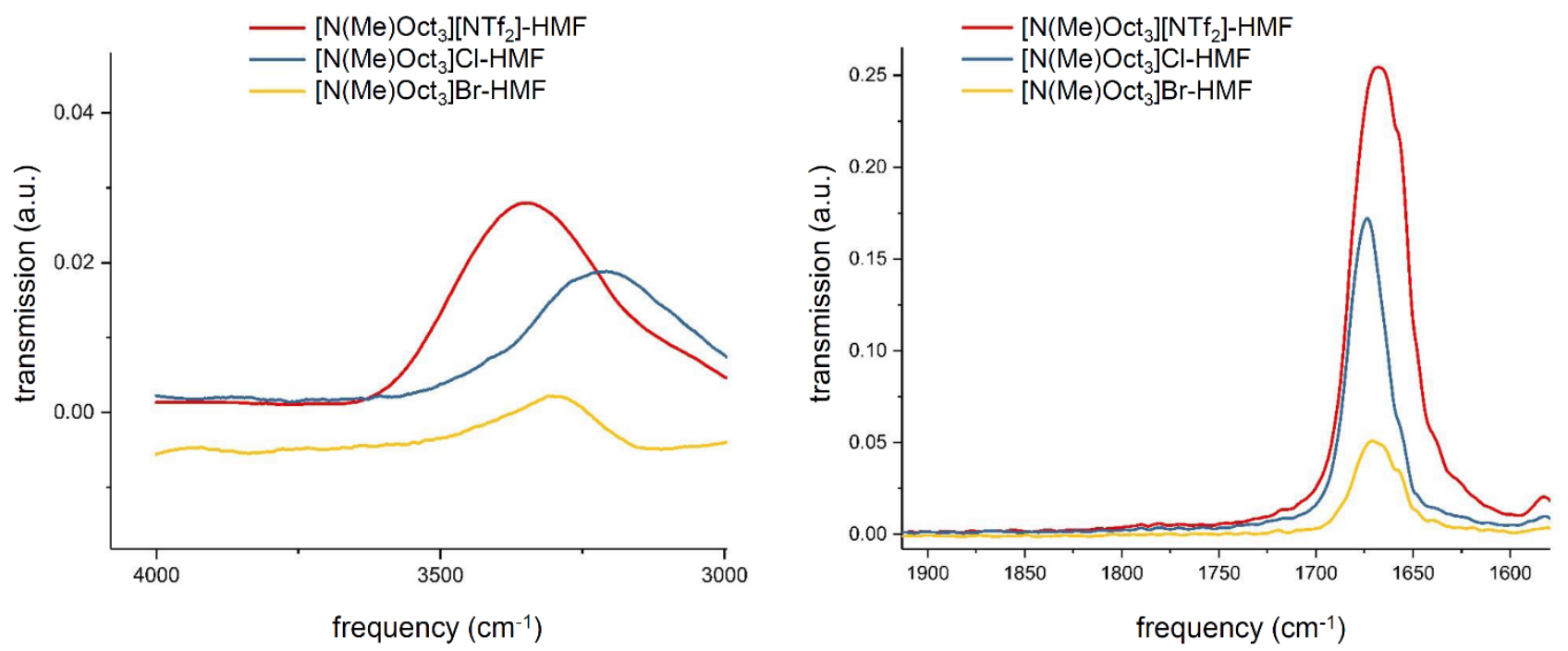

Figure 6. Infrared spectra (in transmission mode) of the hydroxyl and carbonyl features of HMF with anions possessing different hydrogen-bonding ability. 
A comparison between the separation achieved using the $\left[\mathrm{N}(\mathrm{Me}) \mathrm{Oct}_{3}\right]\left[\mathrm{NTf}_{2}\right]$ salt and the chloride analogue is shown in Figure 4. The graph reveals a remarkable improvement for the extraction from [N(Me)Oct 3$]\left[\mathrm{NTf}_{2}\right]$ compared to the chloride analogue, achieving $90 \%$ recovery after only two washes, compared to the 13 needed for the $\left[\mathrm{N}(\mathrm{Me}) \mathrm{Oct}_{3}\right] \mathrm{Cl}$ salt. This also shows that high concentrations of $\mathrm{HMF}(>0.1 \mathrm{M})$ in the water phase can be achieved even at low HMF loadings $(<10 \%)$. In this context, it is worth noting that the efficient synthesis (over $80 \%$ yield) of HMF from fructose in a bistriflimide ionic liquid has been reported by Tian and co-workers using a $\mathrm{VOPO}_{4}$ catalyst. ${ }^{59}$

In order to further confirm the applicability of the system and its potential for recycling, the solubility of the ionic liquids used in the study with water was measured (Table 3).

Table 3. Solubility of hydrophobic ionic liquids in water.

\begin{tabular}{|c|c|}
\hline Ionic Liquid & Solubility (mg IL / $\mathrm{g} \mathrm{H}_{2} \mathrm{O}$ ) \\
\hline$\left[\mathrm{N}(\mathrm{Me}) \mathrm{Oct}_{3}\right] \mathrm{Cl}$ & 2.1 \\
\hline$\left[\mathrm{N}(\mathrm{Me}) \mathrm{Oct}_{3}\right] \mathrm{Br}$ & 0.9 \\
\hline$\left[\mathrm{N}(\mathrm{Me}) \mathrm{Oct}_{3}\right]\left[\mathrm{NTf}_{2}\right]$ & 0.029 \\
\hline$[\mathrm{bmim}]\left[\mathrm{NTf}_{2}\right]$ & 5.5 \\
\hline$\left[\right.$ bmpyr][ $\left.\mathrm{NTf}_{2}\right]$ & 4.7 \\
\hline
\end{tabular}

The $\left[\mathrm{NTf}_{2}\right]^{-}$anions guarantee very low solubility for the three different cations investigated. With the ammonium cation, the solubility is decreased drastically compared to that with the imidazolium and pyridinium cations. Consideration of the high cost associated with imidazolium ionic liquids and losses associated with their greater solubility in water, renders the use of this type of ionic liquid particularly challenging in terms of process economics, requiring a method of recovering the lost ionic liquid from the water phase. In contrast, ammoniumbased ionic liquids have much lower solubility in water but exhibit higher toxicity and viscosity, ${ }^{60}$ necessitating a compromise.

\section{Solvent selection guidelines for product separation}

These results highlight how different scenarios can be optimized for the separation of DFF, FFCA, FDCA, and HMF. These factors need to be considered in order to optimize the synthesis and separation of these molecules in ionic liquid media. The type of catalysis employed for these transformations will influence the system adopted, as heterogeneous catalysis requires good solubility of the final product in the reaction mixture, while homogeneous catalysis conditions necessitate the opposite.

For DFF, the product can be separated easily at any loading by sublimation with the final product being produced in high yield and purity. In this case, either homogeneous or heterogeneous catalysis can be employed with ease.

When isolating FFCA, a large amount of water needs to be added to induce precipitation. For scale up purposes, this translates to excessive energy consumption to dry the IL before re-use. For the synthesis of FFCA, it would be suitable to use homogeneous catalysis conditions in a non-coordinating ionic liquid, such as [bmim][OTf], in which FFCA exhibits low solubility. This will lead to precipitation of the product from solution, allowing simple recovery by filtration.

FDCA can be separated by water addition in the presence of a heterogeneous catalyst or by filtration in [bmim][OTf] operating under homogeneous catalysis conditions.

In the case of HMF, isolation of the product requires multiple water washes when using a chloride-based ionic liquid. Hence, the ideal solvent for the synthesis of this product would be $\left[\mathrm{N}(\mathrm{Me}) \mathrm{Oct}_{3}\right]\left[\mathrm{NTf}_{2}\right]$, which minimizes the number of washes required and guarantees a sufficiently high HMF concentration in the water phase for further transformations (such as conversion to FDCA). The ammonium salt is ideal for homogeneous catalysis, but it can 
prove less efficient under heterogeneous catalysis conditions due to its high viscosity. In this case, the use of imidazolium ionic liquids is recommended.

These results are summarized in Table 4 with reference to the synthesis pathway employed.

Table 4. Solvent design guidelines for the separation of DFF, FFCA, FDCA and HMF from ionic liquid media.

\begin{tabular}{|c|c|c|}
\hline Compound & Homogeneous catalysis & Heterogeneous catalysis \\
\hline DFF & $\begin{array}{l}\text { Sublimation at } 60{ }^{\circ} \mathrm{C} \text { with }[\mathrm{bmim}] \mathrm{Cl} \text { or } \\
{\left[\text { bmim] }\left[\mathrm{NTf}_{2}\right] \text {. Alternatively, filtration from }\right.} \\
{\left[\text { bmim] }\left[\mathrm{NTf}_{2}\right] \text { at room temperature }\right.}\end{array}$ & Sublimation from $[\mathrm{bmim}] \mathrm{Cl}$ or $[\mathrm{bmim}]\left[\mathrm{NTf}_{2}\right]$ \\
\hline FFCA & Precipitation and filtration from [bmim][OTf] & $\begin{array}{l}\text { Water addition to [bmim] Br or [bmim]Cl but } \\
\text { with a high energy cost for drying the IL }\end{array}$ \\
\hline FDCA & Precipitation and filtration from [bmim][OTf] & $\begin{array}{l}\text { Water addition in }[\mathrm{bmim}] \mathrm{Cl} \text { and separation } \\
\text { through filtration }\end{array}$ \\
\hline HMF & $\begin{array}{l}\text { Synthesis in hydrophobic }\left[\mathrm{N}(\mathrm{Me}) \mathrm{Oct}_{3}\right]\left[\mathrm{NTf}_{2}\right] \text {, } \\
\text { followed by washing with water }\end{array}$ & $\begin{array}{l}\text { Synthesis in }[\text { bmim }]\left[\mathrm{NTf}_{2}\right] \text { with water } \\
\text { extraction }\end{array}$ \\
\hline
\end{tabular}

\section{CONCLUSIONS}

This work provides new insights to guide solvent design for ionic liquid based processes. Valuable intermediates were selected that can be synthesized from sugars and HMF and which can be efficiently separated from the ionic liquids. Weak interactions between the aldehyde groups and the anions of the ionic liquid make DFF separation by sublimation extremely efficient from [bmim]Cl and [bmim] $\left[\mathrm{NTf}_{2}\right]$ at low temperature $\left(60^{\circ} \mathrm{C}\right)$ under vacuum, resulting in a product at high purity. FDCA and FFCA can be separated from water-miscible ILs by adding water as an antisolvent. While the energy requirement to regenerate the IL is small in the case of FDCA, excessive water addition is required for FFCA, making the regeneration of the IL extremely energy intensive. The higher solubility of FFCA in ionic liquids can be traced to a synergistic effect between the interaction of water with the aldehyde group and the hydrogen bonding between the $\mathrm{IL}$ anion (e.g. $\left.\mathrm{Cl}^{-}\right)$and the carboxylic acid group. For FFCA, it is therefore recommended that the synthesis is performed in a non-coordinating IL such as [bmim] [OTf]. Hydrophobic, non-coordinating bistriflimide ionic liquids are potential solvents for the generation of HMF due to the possibility of efficient extraction of this product with water. The ammonium-based IL, [N(Me)Oct 3$]\left[N \mathrm{Nf}_{2}\right]$, showed the most favorable partition coefficient (2.28) for HMF, potentially achieving $90 \%$ product recovery with only two washes, minimizing water consumption. As reported previously, HMF present in the aqueous phase can easily be converted to FDCA, which can be subsequently separated from water. It is hoped that the data and conclusions derived from these investigations will be a useful resource for researchers designing systems aimed at the valorization of biomass to furanic building blocks via sugars as intermediates.

\section{ASSOCIATED CONTENT}

Supporting Information is available free of charge at X. Contents include the synthetic procedures and characterization with selected spectra and analytical data. 


\section{AUTHOR INFORMATION}

Corresponding Authors

Jason P. Hallett, E-mail: j.hallett@imperial.ac.uk

James D. E. T. Wilton-Ely, E-mail: j.wilton-ely@imperial.ac.uk

\section{NOTES}

The authors have no conflicts to declare.

\section{ACKNOWLEDGEMENTS}

A.A.G. would like to thank Imperial College London for a President's PhD Scholarship.

\section{REFERENCES}

(1) Huber, G. W.; Iborra, S.; Corma, A. Synthesis of Transportation Fuels from Biomass: Chemistry, Catalysts, and Engineering. Chem. Rev. 2006, 106, 4044-4098, DOI 10.1021/cr068360d.

(2) Constable, D. J. C.; Jimenez-Gonzalez, C.; Henderson, R. K. Perspective on Solvent Use in the Pharmaceutical Industry. Org. Process Res. Dev. 2007, 11, 133-137, DOI 10.1021/op060170h.

(3) Savelski, M. J.; Slater, C. S.; Tozzi, P. V.; Wisniewski, C. M. On the Simulation, Economic Analysis, and Life Cycle Assessment of Batch-Mode Organic Solvent Recovery Alternatives for the Pharmaceutical Industry. Clean Technol. Environ. Policy 2017, 19, 2467-2477, DOI 10.1007/s10098-017-1444-8.

(4) Welton, T. Solvents and Sustainable Chemistry. Proc. R. Soc. A Math. Phys. Eng. Sci. 2015, 471, 1-26. DOI 10.1098/rspa.2015.0502.

(5) Hicks, M. B.; Farrell, W.; Aurigemma, C.; Lehmann, L.; Weisel, L.; Nadeau, K.; Lee, H. Making the Move Towards Modernized Greener Separations: Introduction of the Analytical Method Greenness Score (AMGS) Calculator, Green Chem. 2019, 21, 1816-1826, DOI 10.1039/c8gc03875a.

(6) Plechkova, N. V.; Seddon, K. R. Applications of lonic Liquids in the Chemical Industry. Chem. Soc. Rev. 2008, 37, 123-150, DOI 10.1039/b006677j.

(7) Zhou, L.; He, Y.; Ma, Z.; Liang, R.; Wu, T.; Wu, Y. One-Step Degradation of Cellulose to 5Hydroxymethylfurfural in Ionic Liquid under Mild Conditions. Carbohydr. Polym. 2015, 117, 694-700, DOI 10.1016/j.carbpol.2014.10.062.

(8) Brandt-Talbot, A.; Gschwend, F. J. V.; Fennell, P. S.; Lammens, T. M.; Tan, B.; Weale, J.; Hallett, J. P. An Economically Viable lonic Liquid for the Fractionation of Lignocellulosic Biomass. Green Chem. 2017, 19, 3078-3102, DOI 10.1039/c7gc00705a.

(9) Lopes, M.; Dussan, K.; Leahy, J. J.; da Silva, V. T. Conversion of D-Glucose to 5-Hydroxymethylfurfural Using $\mathrm{Al}_{2} \mathrm{O}_{3}$-Promoted Sulfated Tin Oxide as Catalyst. Catal. Today 2017, 279, 233-243. DOI 10.1016/j.cattod.2016.05.030.

(10) Morales-Leal, F. J.; Rivera de la Rosa, J.; Lucio-Ortiz, C. J.; De Haro-Del Rio, D. A.; Solis Maldonado, C.; Wi, S.; Casabianca, L. B.; Garcia, C. D. Dehydration of Fructose over Thiol- and Sulfonic- Modified 
Alumina in a Continuous Reactor for 5-HMF Production: Study of Catalyst Stability by NMR. Appl. Catal. B 2019, 244, 250-261. DOI 10.1016/J.APCATB.2018.11.053.

(11) Roman-Leshkov, Y.; Chheda, J. N.; Dumesic, J. a. Phase Modifiers Promote Efficient Production of Hydroxymethylfurfural from Fructose. Science 2006, 312, 1933-1937. DOI 10.1126/science.1126337.

Zakrzewska, M. E.; Bogel-łukasik, E.; Bogel-łukasik, R. Ionic Liquid-Mediated Formation of 5Hydroxymethylfurfural as a Promising Biomass-Derived Building Block. Chem. Rev. 2011, 111, 397-417, DOI 10.1021/cr100171a.

(13) Hou, Q.; Zhen, M.; Li, W.; Liu, L.; Liu, J.; Zhang, S.; Nie, Y.; Bai, C.; Bai, X.; Ju, M. Efficient Catalytic Conversion of Glucose into 5-Hydroxymethylfurfural by Aluminum Oxide in Ionic Liquid. Appl. Catal. B 2019, 253, 1-10. DOI 10.1016/j.apcatb.2019.04.003.

(14) Zhao, H., Holladay, J.E., Brown, H., Zhang, Z. C. Metal Chlorides in Ionic Liquid Solvents Convert Sugar to 5-Hydroxymethyfurfural. Science 2007, 316, 1597-1600. DOI 10.1126/science.1141199.

(15) Al Ghatta, A.; Wilton-Ely, J. D. E. T.; Hallett, J. P. Rapid, High-Yield Fructose Dehydration to HMF in the Ionic Liquid [Bmim][OTf]. ChemSusChem 2019. DOI 10.1002/cssc.201901529.

(16) Eminov, S.; Wilton-Ely, J. D. E. T.; Hallett, J. P. Highly Selective and Near-Quantitative Conversion of Fructose to 5-Hydroxymethylfurfural Using Mildly Acidic lonic Liquids. ACS Sustain. Chem. Eng. 2014, 2, 978-981. DOI 10.1021/sc400553q.

(17) Van Putten, R.-J. J.; van der Waal, J. C.; de Jong, E.; Rasrendra, C. B.; Heeres, H. J.; de Vries, J. G. Hydroxymethylfurfural, a Versatile Platform Chemical Made from Renewable Resources. Chem. Rev. 2013, 113, 1499-1597, DOI 10.1021/cr300182k.

(18) Wang, H.; Zhu, C.; Li, D.; Liu, Q.; Tan, J.; Wang, C.; Cai, C.; Ma, L. Recent Advances in Catalytic Conversion of Biomass to 5-Hydroxymethylfurfural and 2,5-Dimethylfuran. Renew. Sustain. Energy Rev. 2019, 103, 227-247, DOI 10.1016/j.rser.2018.12.010.

(19) Wei, Z.; Liu, Y.; Thushara, D.; Ren, Q. Entrainer-Intensified Vacuum Reactive Distillation Process for the Separation of 5-Hydroxylmethylfurfural from the Dehydration of Carbohydrates Catalyzed by a Metal SaltIonic Liquid. Green Chem. 2012, 14, 1220-1226, DOI 10.1039/c2gc16671b.

(20) Shi, C.; Xin, J.; Liu, X.; Lu, X.; Zhang, S. Using Sub/Supercritical $\mathrm{CO}_{2}$ as "Phase Separation Switch" for the Efficient Production of 5-Hydroxymethylfurfural from Fructose in an Ionic Liquid/Organic Biphasic System. ACS Sustain. Chem. Eng. 2016, 4, 557-563, DOI 10.1021/acssuschemeng.5b00889.

(21) Sun, X.; Liu, Z.; Xue, Z.; Zhang, Y.; Mu, T. Extraction of 5-HMF from the Conversion of Glucose in lonic Liquid $[\mathrm{bmim}] \mathrm{Cl}$ by Compressed Carbon Dioxide. Green Chem. 2015, 17, 2719-2722, DOI $10.1039 / \mathrm{c} 5 \mathrm{gc00092k}$.

(22) Hou, Q.; Li, W.; Zhen, M.; Liu, L.; Chen, Y.; Yang, Q.; Huang, F.; Zhang, S.; Ju, M. An lonic Liquid-Organic Solvent Biphasic System for Efficient Production of 5-Hydroxymethylfurfural from Carbohydrates at High Concentrations. RSC Adv. 2017, 7, 47288-47296, DOI 10.1039/c7ra10237b.

(23) Zhang, S.; Li, W.; Zeng, X.; Lin, L. Recent Progress and Prospects in the Selective Catalytic Production of 2,5-Diformylfuran. J. Bioprocess Eng. Biorefinery 2014, 3, 23-34, DOI 10.1166/jbeb.2014.1072.

(24) Ventura, M.; Aresta, M.; Dibenedetto, A. Selective Aerobic Oxidation of 5-(Hydroxymethyl)furfural to 5Formyl-2-furancarboxylic Acid in Water. ChemSusChem 2016, 9, 1096-1100, DOI 10.1002/cssc.201600060.

(25) Sajid, M.; Zhao, X.; Liu, D. Production of 2,5-Furandicarboxylic Acid (FDCA) from 5-Hydroxymethylfurfural (HMF): Recent Progress Focusing on the Chemical-Catalytic Routes. Green Chem. 2018, 20, 5427-5453, DOI 10.1039/c8gc02680g. 
Pérez-Bustos, H. F.; Lucio-Ortiz, C. J.; de la Rosa, J. R.; de Haro del Río, D. A.; Sandoval-Rangel, L.; Martínez-Vargas, D. X.; Maldonado, C. S.; Rodriguez-González, V.; Garza-Navarro, M. A.; Morales-Leal, F. J. Synthesis and Characterization of Bimetallic Catalysts Pd-Ru and Pt-Ru Supported on Y-Alumina and Zeolite FAU for the Catalytic Transformation of HMF. Fuel 2019, 239, 191-201. DOI 10.1016/J.FUEL.2018.10.001.

(27) Li, Y.; Li, Y.; Li, P.; Cao, P.; Wang, X.; Guan, H. POM@surf(n)/CeO 2 Electrospun Nanofibers for the Facile Oxidation of 5-HMF to DFF. Appl. Catal. A 2019, 583, 117-122. DOI 10.1016/j.apcata.2019.117122.

(28) Zhao, J.; Jayakumar, A.; Hu, Z. T.; Yan, Y.; Yang, Y.; Lee, J. M. $\mathrm{MoO}_{3}$-Containing Protonated Nitrogen Doped Carbon as a Bifunctional Catalyst for One-Step Synthesis of 2,5-Diformylfuran from Fructose. ACS Sustain. Chem. Eng. 2018, 6, 284-291. DOI 10.1021/acssuschemeng.7b02408.

(29) Wang, Q.; Hou, W.; Meng, T.; Hou, Q.; Zhou, Y.; Wang, J. Direct Synthesis of 2,5-Diformylfuran from Carbohydrates via Carbonizing Polyoxometalate Based Mesoporous Poly(lonic Liquid). Catal. Today 2019, 319, 57-65. DOI 10.1016/j.cattod.2018.07.042.

(30) Zhang, W.; Meng, T.; Tang, J.; Zhuang, W.; Zhou, Y.; Wang, J. Direct Synthesis of 2,5-Diformylfuran from Carbohydrates Using High-Silica MOR Zeolite-Supported Isolated Vanadium Species. ACS Sustain. Chem. Eng. 2017, 5, 10029-10037. DOI 10.1021/acssuschemeng.7b02002.

(31) Antonyraj, C. A.; Jeong, J.; Kim, B.; Shin, S.; Kim, S.; Lee, K. Y.; Cho, J. K. Selective Oxidation of HMF to DFF Using Ru/Alumina Catalyst in Moderate Boiling Solvents toward Industrial Production. J. Ind. Eng. Chem. 2013, 19, 1056-1059. DOI 10.1016/j.jiec.2012.12.002.

Zhou, X.; Song, K.; Li, Z.; Kang, W.; Ren, H.; Su, K.; Zhang, M.; Cheng, B. The Excellent Catalyst Support of $\mathrm{Al}_{2} \mathrm{O}_{3}$ Fibers with Needle-like Mullite Structure and HMF Oxidation into FDCA over $\mathrm{CuO} / \mathrm{Al}_{2} \mathrm{O}_{3}$ Fibers. Ceram. Int. 2019, 45, 2330-2337. DOI 10.1016/J.CERAMINT.2018.10.148.

(33) Yi, G.; Teong, S. P.; Zhang, Y. Base-Free Conversion of 5-Hydroxymethylfurfural to 2,5-Furandicarboxylic Acid over a Ru/C Catalyst. Green Chem. 2016, 18, 979-983. DOI 10.1039/C5GC01584G.

(34) Zhang, C.; Chang, X.; Zhu, L.; Xing, Q.; You, S.; Qi, W.; Su, R.; He, Z. Highly Efficient and Selective Production of FFCA from CotA-TJ102 Laccase-Catalyzed Oxidation of 5-HMF. Int. J. Biol. Macromol. 2019, 128, 132-139. DOI 10.1016/j.jijbiomac.2019.01.104.

(35) Chen, R.; Xin, J.; Yan, D.; Dong, H.; Lu, X.; Zhang, S. Highly Efficient Oxidation of 5-Hydroxymethylfurfural to 2,5-Furandicarboxylic Acid with Heteropoly Acids. ChemSusChem 2019, DOI 10.1002/cssc.201900651.

(36) Yan, D.; Wang, G.; Gao, K.; Lu, X.; Xin, J.; Zhang, S. One-Pot Synthesis of 2,5-Furandicarboxylic Acid from Fructose in lonic Liquids. Ind. Eng. Chem. Res. 2018, 57, 1851-1858, DOI 10.1021/acs.iecr.7b04947.

(37) Yan, D.; Xin, J.; Zhao, Q.; Gao, K.; Lu, X.; Wang, G.; Zhang, S. Fe-Zr-O Catalyzed Base-Free Aerobic Oxidation of 5-HMF to 2,5-FDCA as a Bio-Based Polyester Monomer. Catal. Sci. Technol. 2018, 8, 164175, DOI 10.1039/c7cy01704a.

(38) Gajula, S.; Inthumathi, K.; Arumugam, S. R.; Srinivasan, K. Strategic Designing on Selection of Solvent Systems for Conversion of Biomass Sugars to Furan Derivatives and Their Separation. ACS Sustain. Chem. Eng. 2017, 5, 5373-5381, DOI 10.1021/acssuschemeng.7b00681.

(39) Chen, G.; Wu, L.; Fan, H.; Li, B. G. Highly Efficient Two-Step Synthesis of 2,5-Furandicarboxylic Acid from Fructose without 5-Hydroxymethylfurfural (HMF) Separation: In Situ Oxidation of HMF in Alkaline Aqueous $\mathrm{H}_{2} \mathrm{O}$ /DMSO Mixed Solvent under Mild Conditions. Ind. Eng. Chem. Res. 2018, 57, 16172-16181, DOI 10.1021/acs.iecr.8b03589.

(40) Thananatthanachon, T.; Rauchfuss, T. B. Efficient Route to Hydroxymethylfurans from Sugars via Transfer Hydrogenation. ChemSusChem 2010, 3, 1139-1141, DOI 10.1002/cssc.201000209. 

with Solid Acid Catalysts by Simple Water Removal Methods. Catal. Commun. 2009, 10, 1849-1853, DOI 10.1016/j.catcom.2009.06.012.

(42) Lv, G.; Wang, H.; Yang, Y.; Li, X.; Deng, T.; Chen, C.; Zhu, Y.; Hou, X. Aerobic Selective Oxidation of 5Hydroxymethyl-Furfural over Nitrogen-Doped Graphene Materials with 2,2,6,6-Tetramethylpiperidin-Oxyl as Co-Catalyst. Catal. Sci. Technol. 2016, 6, 2377-2386, DOI 10.1039/c5cy01149c.

(43) Halliday, G. A.; Young, R. J.; Grushin, V. V. One-Pot, Two-Step, Practical Catalytic Synthesis of 2,5Diformylfuran from Fructose. Org. Lett. 2003, 5, 2003-2005. DOI 10.1021/ol034572a.

(44) Ferro, V. R.; Moya, C.; Moreno, D.; Santiago, R.; De Riva, J.; Pedrosa, G.; Larriba, M.; Diaz, I.; Palomar, J. Enterprise lonic Liquids Database (ILUAM) for Use in Aspen ONE Programs Suite with COSMO-Based Property Methods. Ind. Eng. Chem. Res. 2018, 57, 980-989, DOI 10.1021/acs.iecr.7b04031.

(45) Hu, L.; Sun, Y.; Lin, L. Efficient Conversion of Glucose into 5-Hydroxymethylfurfural by Chromium(III) Chloride in Inexpensive lonic Liquid. Ind. Eng. Chem. Res. 2012, 51, 1099-1104, DOI 10.1021/ie202174f.

(46) Xu, J.; Paimin, R.; Shen, W.; Wang, X. An Investigation of Solubility of Aliquat 336 in Different Extracted Solutions. Fibers Polym. 2003, 4, 27-31, DOI 10.1007/BF02899326.

(47) Zhou, J.; Xia, Z.; Huang, T.; Yan, P.; Xu, W.; Xu, Z.; Wang, J.; Zhang, Z. C. An lonic Liquid-Organics-Water Ternary Biphasic System Enhances the 5-Hydroxymethylfurfural Yield in Catalytic Conversion of Glucose at High Concentrations. Green Chem. 2015, 17, 4206-4216. DOI 10.1039/c5gc01039j.

(48) Rutz, C.; Schmolke, L.; Gvilava, V.; Janiak, C. Anion Analysis of Ionic Liquids and lonic Liquid Purity Assessment by Ion Chromatography. Z. Anorg. Allg. Chem. 2017, 643, 130-135, DOI 10.1002/zaac.201600437.

(49) Wang, H.; Liu, S.; Zhao, Y.; Zhang, H.; Wang, J. Molecular Origin for the Difficulty in Separation of 5Hydroxymethylfurfural from Imidazolium Based Ionic Liquids. ACS Sustain. Chem. Eng. 2016, 4, 67126721, DOI 10.1021/acssuschemeng.6b01652.

(50) Hussain Motagamwala, A.; Won, W.; Sener, C.; Alonso, D. M.; Maravelias, C. T.; Dumesic, J. A. Toward Biomass-Derived Renewable Plastics: Production of 2,5-Furandicarboxylic Acid from Fructose. Sci. Adv. 2018, 4, 1-8. DOI 10.1126/sciadv.aap9722.

(51) Dijkman, W. P.; Groothuis, D. E.; Fraaije, M. W. Enzyme-Catalyzed Oxidation of 5-Hydroxymethylfurfural to Furan-2,5-dicarboxylic Acid. Angew. Chem. Int. Ed. 2014, 53, 6515-6518, DOI 10.1002/anie.201402904.

(52) Davis, S. E.; Zope, B. N.; Davis, R. J. On the Mechanism of Selective Oxidation of 5-Hydroxymethylfurfural to 2,5-Furandicarboxylic Acid over Supported Pt and Au Catalysts. Green Chem. 2012, 14, 143-147. DOI 10.1039/C1GC16074E.

(53) Chen, Z.; Yang, D. Determination of Mutual Solubility between n-Alkanes/n-Alkylbenzenes and Water by Using Peng-Robinson Equation of State with Modified Alpha Functions and Generalized BIP Correlations. Fluid Phase Equilib. 2018, 477, 19-29. DOI 10.1016/j.fluid.2018.08.010.

(54) Rivera de la Rosa, J.; Elizondo-Martínez, P.; Nájera Martínez, B.; Pérez Rodríguez, N.; Rodríguez Díaz, J. Determination of the Solubility of Copper with Three Commercial Ligands and One Novel Ligand in Supercritical $\mathrm{CO}_{2}$, Mater. Manuf. Processes, 2011, 26, 304-310. DOI 10.1080/10426914.2010.544807.

(55) Eminov, S.; Filippousi, P.; Brandt, A.; Wilton-Ely, J.; Hallett, J. Direct Catalytic Conversion of Cellulose to 5-Hydroxymethylfurfural Using lonic Liquids. Inorganics 2016, 4, 1-15, DOI 10.3390/inorganics4040032.

(56) Ilgen, F.; Ott, D.; Kralisch, D.; Reil, C.; Palmberger, A.; König, B. Conversion of Carbohydrates into 5Hydroxymethylfurfural in Highly Concentrated Low Melting Mixtures. Green Chem. 2009, 11, 1948-1954, DOI 10.1039/b917548m. 
(57) Li, Y. N.; Wang, J. Q.; He, L. N.; Yang, Z. Z.; Liu, A. H.; Yu, B.; Luan, C. R. Experimental and Theoretical Studies on Imidazolium Ionic Liquid-Promoted Conversion of Fructose to 5-Hydroxymethylfurfural. Green Chem. 2012, 14, 2752-2758, DOI 10.1039/c2gc35845j.

(58) Li, C.; Zhao, Z. K.; Wang, A.; Zheng, M.; Zhang, T. Production of 5-Hydroxymethylfurfural in lonic Liquids under High Fructose Concentration Conditions. Carbohydr. Res. 2010, 345, 1846-1850, DOI 10.1016/j.carres.2010.07.003.

(59) Tian, C.; Zhu, X.; Chai, S. H.; Wu, Z.; Binder, A.; Brown, S.; Li, L.; Luo, H.; Guo, Y.; Dai, S. Three-Phase Catalytic System of $\mathrm{H}_{2} \mathrm{O}$, Ionic Liquid, and $\mathrm{VOPO}_{4}-\mathrm{SiO}_{2}$ Solid Acid for Conversion of Fructose to 5Hydroxymethylfurfural. ChemSusChem 2014, 7, 1703-1709, DOI 10.1002/cssc.201400119.

(60) Berglind, R.; Dave, G. Influence of a Co-Solvent (Acetone) and Ultrasonication on the Acute Toxicity of a Quaternary Amine (Aliquat 336) and an Organophosphorus Compound (HDEHP) to Daphnia Magna. Bull. Environ. Contam. Toxicol. 1981, 27, 316-325, DOI 10.1007/BF01611026. 

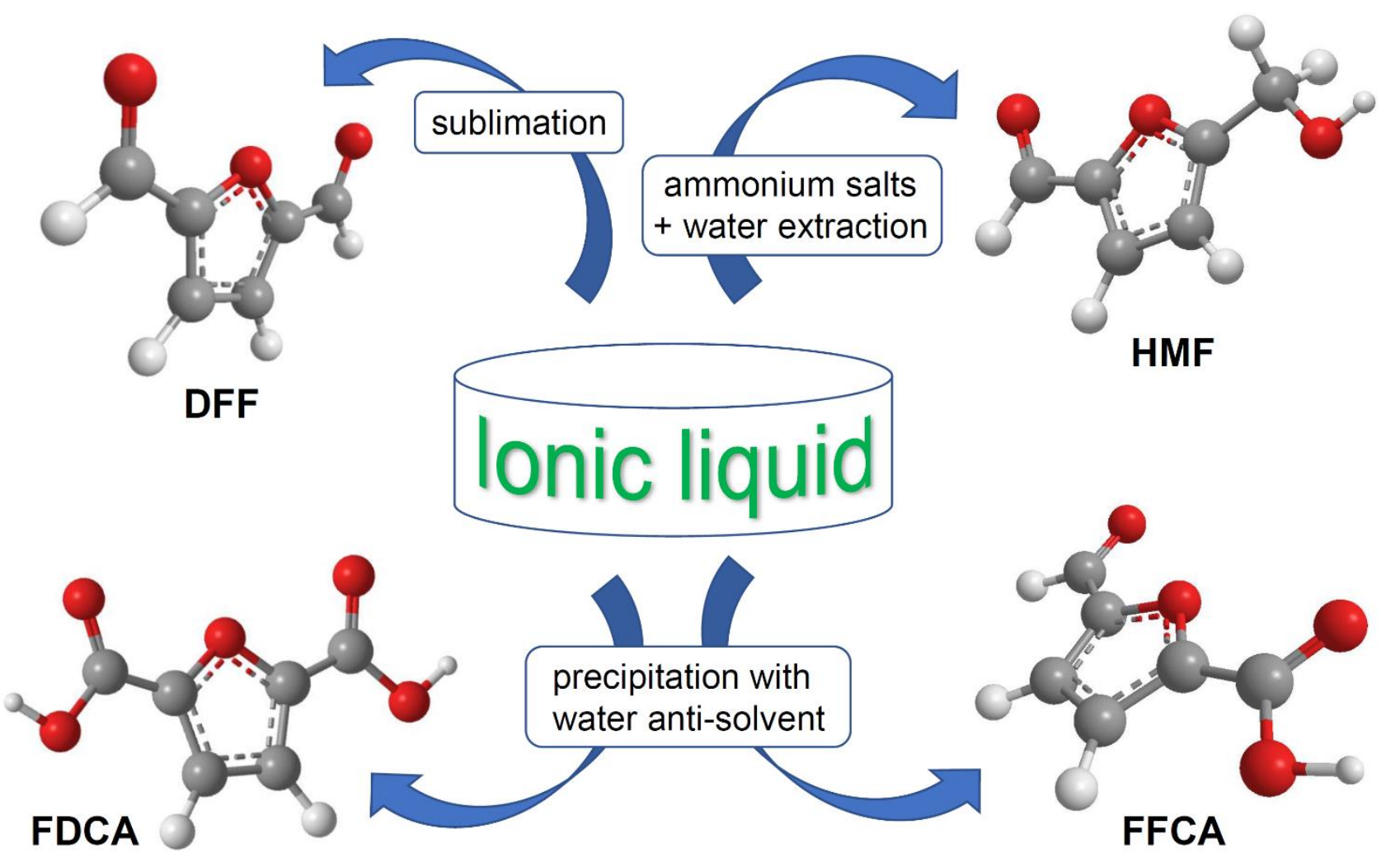

HMF

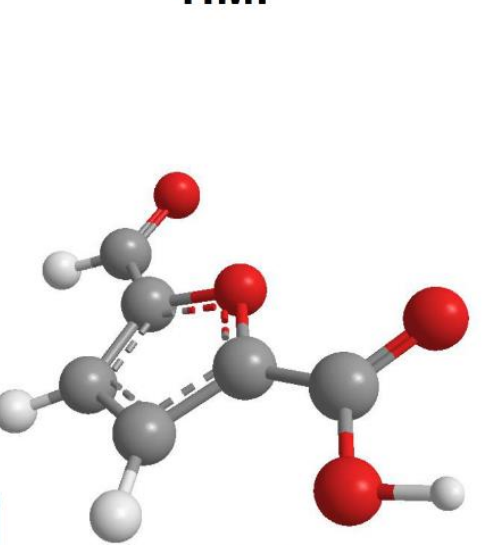

FFCA

Solvent design strategies to guide the selective synthesis, separation and purification of furanic compounds from sugars in ionic liquids. 


\section{Strategies for the Separation of the Furanic Compounds HMF, DFF, FFCA and FDCA from Ionic Liquids}

Amir Al Ghatta, James D. E. T. Wilton Ely* and Jason P. Hallett*

Department of Chemical Engineering, Imperial College, South Kensington Campus, London SW7 2AZ.

Department of Chemistry, Imperial College, Molecular Sciences Research Hub, White City Campus, London W12 OBZ.

S1 General considerations page $\mathrm{S} 2$

S2 Synthesis of ionic liquids page $\mathrm{S} 2$

S2.1 Synthesis of [bmim] Cl, [bmim]Br and [bmpyr][NTf 2$]$ page $\mathrm{S} 2$

S2.2 Synthesis of [bmim][OTf], [bmim][NTf 2 and [bmpyr][NTf $\mathrm{NT}_{2}$ page $S 7$

S3 Aspen Simulation page $S 13$

S4 Sublimation of DFF page $\mathrm{S} 14$

S5 Numerical data page $S 18$

S6 References page $\$ 20$

Number of figures: 27

Number of tables: 9 


\section{S1. General considerations}

Petroleum ether refers to the fraction boiling in the range $40-60{ }^{\circ} \mathrm{C}$. Electrospray mass spectra were obtained using a Synapt G2 instrument (in positive mode). Nuclear magnetic resonance (NMR) spectroscopy was performed using a Bruker $400 \mathrm{MHz}$ spectrometer using $\mathrm{CDCl}_{3}$ or deuterated DMSO as solvent. All couplings are reported in Hertz.

\section{S2. Synthesis of ionic liquids}

\section{S2.1 Synthesis of [bmim] Cl and [bmim]Br}

[bmim] Cl was synthesized on a large scale $(350 \mathrm{~g})$. Manipulation of all chemicals was performed by cannulation under inert conditions. 1-methylimidazole $(160 \mathrm{~mL}, 2 \mathrm{~mol})$ was transferred to a three neck flask equipped with an air condenser followed by the addition of acetonitrile $(100 \mathrm{~mL})$. The mixture was heated at $60{ }^{\circ} \mathrm{C}$ under stirring and chlorobutane (230 $\mathrm{mL}, 2.2 \mathrm{~mol}, 1.1 \mathrm{eq})$ was then added dropwise using a dropping funnel. The reaction mixture was heated for 3 days at $70^{\circ} \mathrm{C}$. Complete consumption of 1 -methylimidazole was confirmed by ${ }^{1} \mathrm{H}$ NMR spectroscopy. [bmim] $\mathrm{Cl}$ was precipitated by gradual addition of ethyl acetate at 3 ${ }^{\circ} \mathrm{C}$. The resultant white crystals were washed under inert conditions with portions of ethyl acetate ( $2 \mathrm{~L}$ in total), dried under vacuum and stored in a glovebox.

[bmim] $\mathrm{Br}$ was synthesized by the same procedure using bromobutane and [bmpyr]Cl was prepared in the same way using $\mathrm{N}$-methylpyrrolidine. Data for $[\mathrm{bmim}] \mathrm{Cl},[\mathrm{bmim}] \mathrm{Br}$ and [bmpyr] $\mathrm{Cl}$ were in good agreement with those reported previously. ${ }^{\mathrm{S} 1, \mathrm{~S} 2, \mathrm{S3}}$

[bmim]Cl: ${ }^{1} \mathrm{H}$ NMR (DMSO-d $\left.\mathrm{d}_{6}\right): \delta 9.62\left(\mathrm{~s}, 1 \mathrm{H}, \mathrm{N}_{2} \mathrm{CH}\right), 7.92,7.84(\mathrm{~m} \times 2,2 \times 1 \mathrm{H}, 2 \times \mathrm{N}-\mathrm{CH})$, $4.20\left(\mathrm{t}, 2 \mathrm{H}, \mathrm{N}-\mathrm{CH}_{2}-\mathrm{CH}_{2}, J_{\mathrm{HH}}=7.2 \mathrm{~Hz}\right), 3.88\left(\mathrm{~s}, 3 \mathrm{H}, \mathrm{N}-\mathrm{CH}_{3}\right), 1.77,1.24\left(\mathrm{~m} \times 2,2 \times 2 \mathrm{H}, \mathrm{N}-\mathrm{CH}_{2}-\right.$ $\mathrm{C}_{2}-\mathrm{C}_{2}-\mathrm{CH}_{3}$ ), 0.87 (t, 3H, $-\mathrm{CH}_{2}-\underline{\mathrm{C}}_{3}, J_{\mathrm{HH}}=7.3 \mathrm{~Hz}$ ) ppm. ${ }^{13} \mathrm{C}\left\{{ }^{1} \mathrm{H}\right\}$ NMR (DMSO-d $\mathrm{d}_{6}$ ): $\delta 137.2$ $\left(\mathrm{N}_{2}-\mathrm{CH}\right), 124.1,122.7(2 \times \mathrm{N}-\mathrm{CH}), 48.8\left(\mathrm{~N}-\underline{\mathrm{CH}_{2}}-\mathrm{CH}_{2}-\mathrm{CH}_{2}-\mathrm{CH}_{3}\right), 36.14\left(\mathrm{NCH}_{3}\right), 31.8$ $\left(\mathrm{NCH}_{2} \underline{\mathrm{C}} \mathrm{H}_{2} \mathrm{CH}_{2} \mathrm{CH}_{3}\right), 19.2\left(\mathrm{NCH}_{2}-\mathrm{CH}_{2}-\mathrm{CH}_{2}-\mathrm{CH}_{3}\right), 13.7\left(\mathrm{~N}-\left(\mathrm{CH}_{2}\right)_{2}-\mathrm{CH}_{2}-\underline{\mathrm{CH}_{3}}\right)$ ppm. MS (ES, +ve mode) $: m / z$ (abundance) $=139(100)[\text { bmim }]^{+}$.

[bmim]Br: ${ }^{1} \mathrm{H}$ NMR (DMSO-d 6 ): $\delta 9.33\left(\mathrm{~s}, 1 \mathrm{H}, \mathrm{N}_{2} \mathrm{CH}\right), 7.86,7.78(\mathrm{~m} \times 2,2 \times 1 \mathrm{H}, 2 \times \mathrm{N}-\mathrm{CH})$, $4.20\left(\mathrm{t}, 2 \mathrm{H}, \mathrm{N}-\mathrm{CH}_{2}-\mathrm{CH}_{2}, J_{H H}=7.2 \mathrm{~Hz}\right), 3.87\left(\mathrm{~s}, 3 \mathrm{H}, \mathrm{N}-\mathrm{CH}_{3}\right), 1.76,1.25\left(\mathrm{~m} \times 2,2 \times 2 \mathrm{H}, \mathrm{N}-\mathrm{CH}_{2}-\right.$ $\mathrm{C}_{2}-\mathrm{C}_{2}-\mathrm{CH}_{3}$ ), 0.88 (t, 3H, $-\mathrm{CH}_{2}-\underline{C}_{3}, J_{H H}=7.3 \mathrm{~Hz}$ ) ppm. ${ }^{13} \mathrm{C}\left\{{ }^{1} \mathrm{H}\right\} \mathrm{NMR}$ (DMSO-d $)$ ): $\delta 137.2$ $\left(\mathrm{N}_{2}-\mathrm{CH}\right), \quad 124.1,122.7(2 \times \mathrm{N}-\mathrm{CH}), 48.8\left(\mathrm{~N}-\underline{\mathrm{CH}_{2}}-\mathrm{CH}_{2}-\mathrm{CH}_{2}-\mathrm{CH}_{3}\right), 36.14\left(\mathrm{NCH}_{3}\right), 31.8$ $\left(\mathrm{NCH}_{2} \underline{\mathrm{C}} \mathrm{H}_{2} \mathrm{CH}_{2} \mathrm{CH}_{3}\right), 19.2\left(\mathrm{NCH}_{2}-\mathrm{CH}_{2}-\underline{\mathrm{CH}_{2}}-\mathrm{CH}_{3}\right), 13.7\left(\mathrm{~N}-\left(\mathrm{CH}_{2}\right)_{2}-\mathrm{CH}_{2}-\underline{\mathrm{C}} \mathrm{H}_{3}\right)$ ppm. MS (ES, +ve mode): $m / z$ (abundance) $=357(31)\left[(\text { bmim })_{2} \mathrm{Br}\right]^{+}, 139(100)[\mathrm{bmim}]^{+}$.

[bmpyr]Cl: ${ }^{1} \mathrm{H}$ NMR (DMSO-d $\left.\mathrm{d}_{6}\right)$ : 3.58-3.39 (m, 6H, 2,5-pyrrole- $\mathrm{CH}_{2}$ and $\mathrm{N}-\mathrm{CH}_{2}-\mathrm{CH}_{2}-\mathrm{CH}_{2}-$ $\left.\mathrm{CH}_{3}\right), 3.03\left(\mathrm{~s}, 3 \mathrm{H}, \mathrm{N}-\mathrm{CH}_{3}\right), 2.02-2.05\left(\mathrm{~m}, 2 \times 2 \mathrm{H}, 3,4-\right.$ pyrrole- $\left.\mathrm{CH}_{2}\right), 1.65(\mathrm{~m}, 2 \mathrm{H}$, $\left.\mathrm{NCH}_{2} \mathrm{C}_{2} \mathrm{CH}_{2} \mathrm{CH}_{3}, J_{\mathrm{HH}}=7.2 \mathrm{~Hz}\right), 1.2\left(\mathrm{~m}, 2 \mathrm{H}, \mathrm{NCH}_{2} \mathrm{CH}_{2} \mathrm{C}_{2} \mathrm{CH}_{3}, J_{\mathrm{HH}}=7.3 \mathrm{~Hz}\right), 0.86(\mathrm{t}, 3 \mathrm{H}, \mathrm{N}-$ $\left.\mathrm{CH}_{2}-\mathrm{CH}_{2}-\mathrm{CH}_{2}-\underline{\mathrm{C}}_{3}, J_{\mathrm{HH}}=7.3 \mathrm{~Hz}\right)$ ppm. ${ }^{13} \mathrm{C}\left\{{ }^{1} \mathrm{H}\right\} \mathrm{NMR}\left(\mathrm{DMSO}-\mathrm{d}_{6}\right): 63.7\left(\mathrm{~s}, \mathrm{~N}-\underline{\mathrm{C}} \mathrm{H}_{2}-\mathrm{CH}_{2}\right.$ ), 63.0 


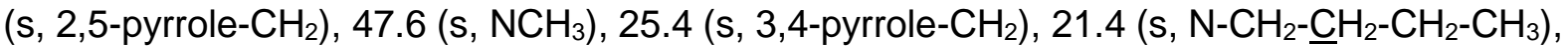
$19.8\left(\mathrm{~s}, \mathrm{~N}-\left(\mathrm{CH}_{2}\right)_{2}-\underline{\mathrm{C}} \mathrm{H}_{2}-\mathrm{CH}_{3}\right), 14.0\left(\mathrm{~s}, \mathrm{~N}-\left(\mathrm{CH}_{2}\right)_{3}-\underline{\mathrm{C}} \mathrm{H}_{3}\right) . \mathrm{m} / \mathrm{z}$ (abundance) = 142(100) [bmpyr].

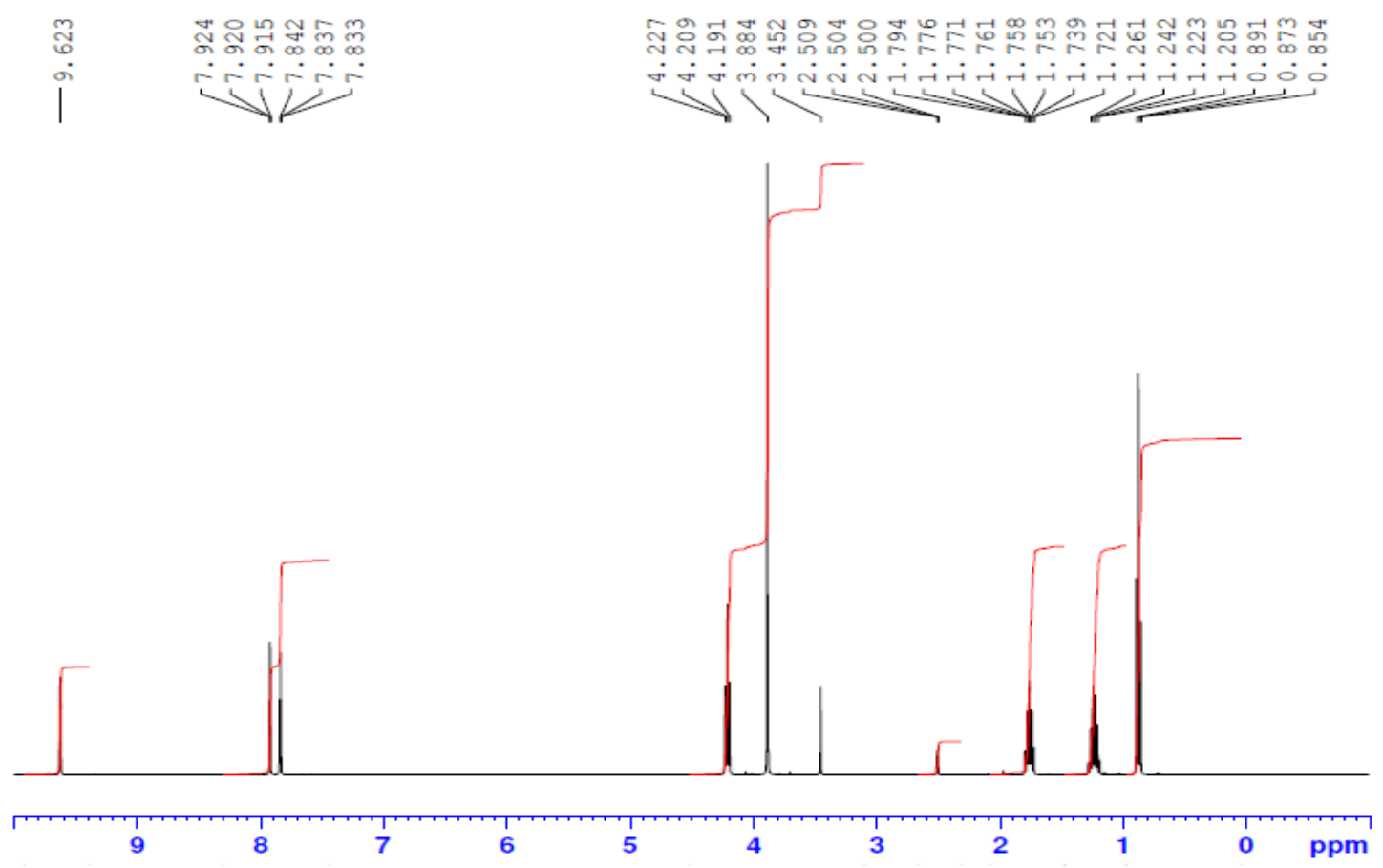

Figure $\mathrm{S} 1{ }^{1} \mathrm{H}$ NMR spectrum of [bmim] $\mathrm{Cl}$ in deuterated DMSO.

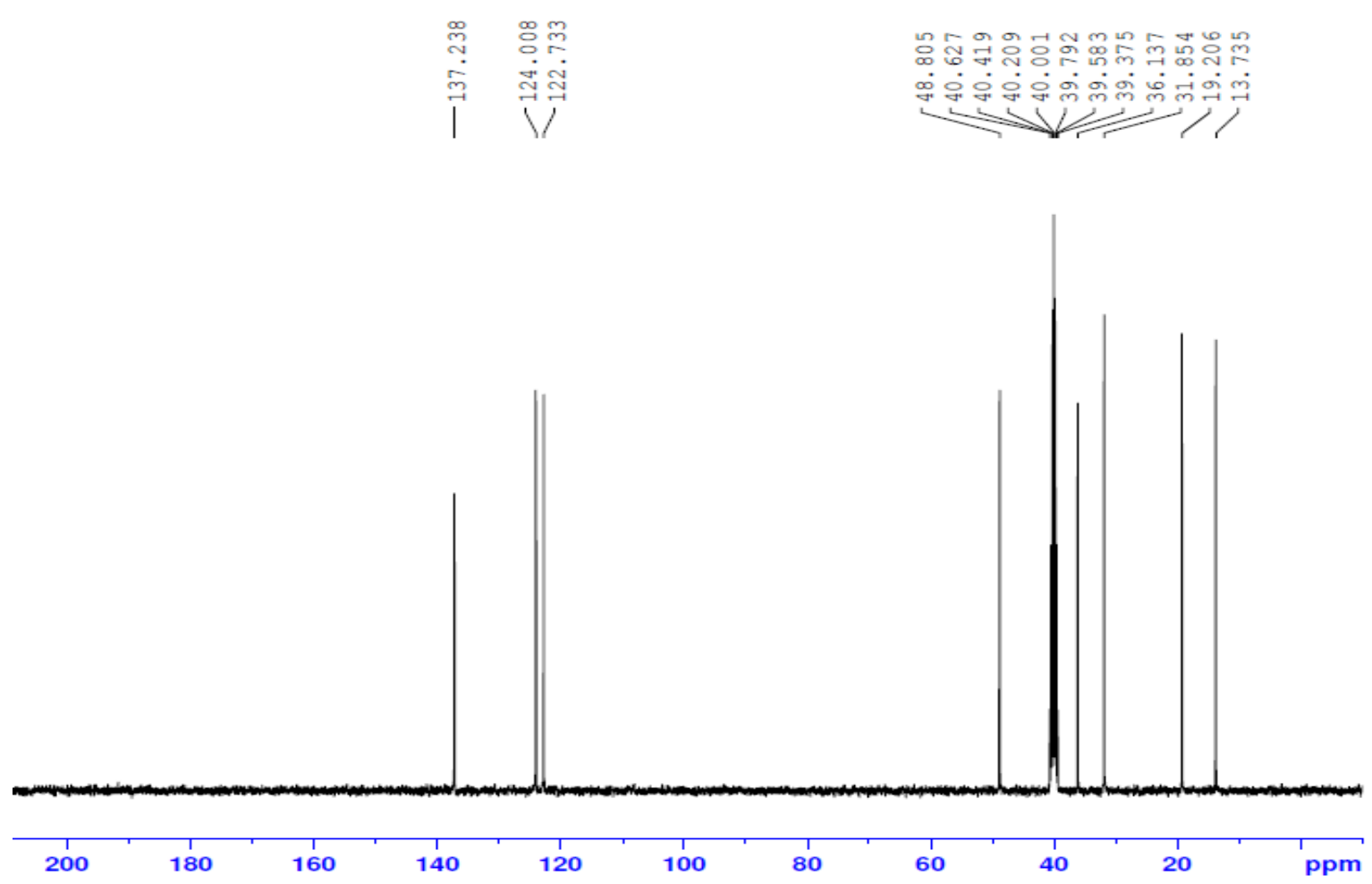

Figure $\mathbf{S 2}{ }^{13} \mathrm{C}\left\{{ }^{1} \mathrm{H}\right\}$ NMR spectrum of [bmim] Cl in deuterated DMSO. 


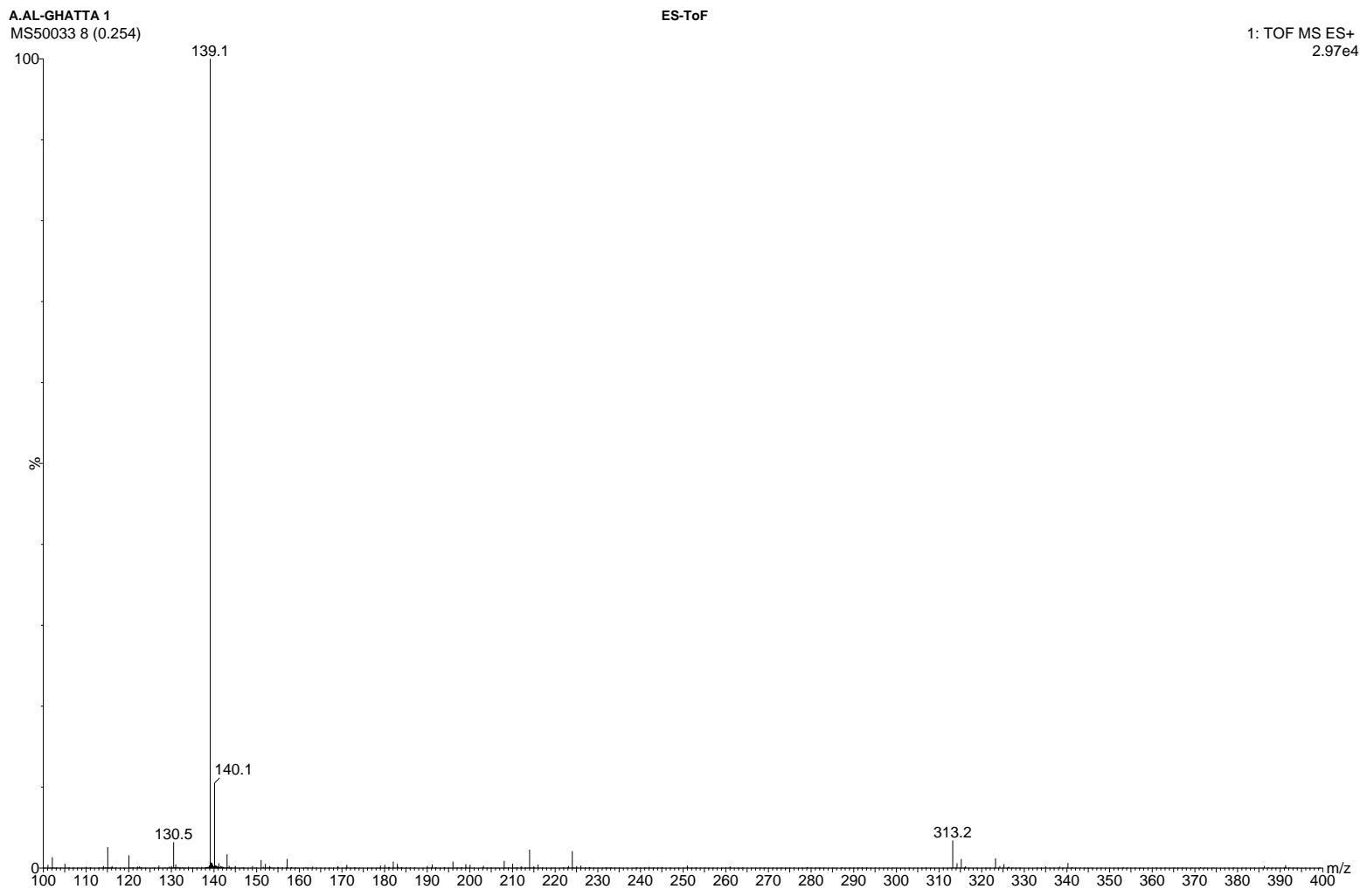

Figure S3 Mass spectrum of [bmim] Cl.

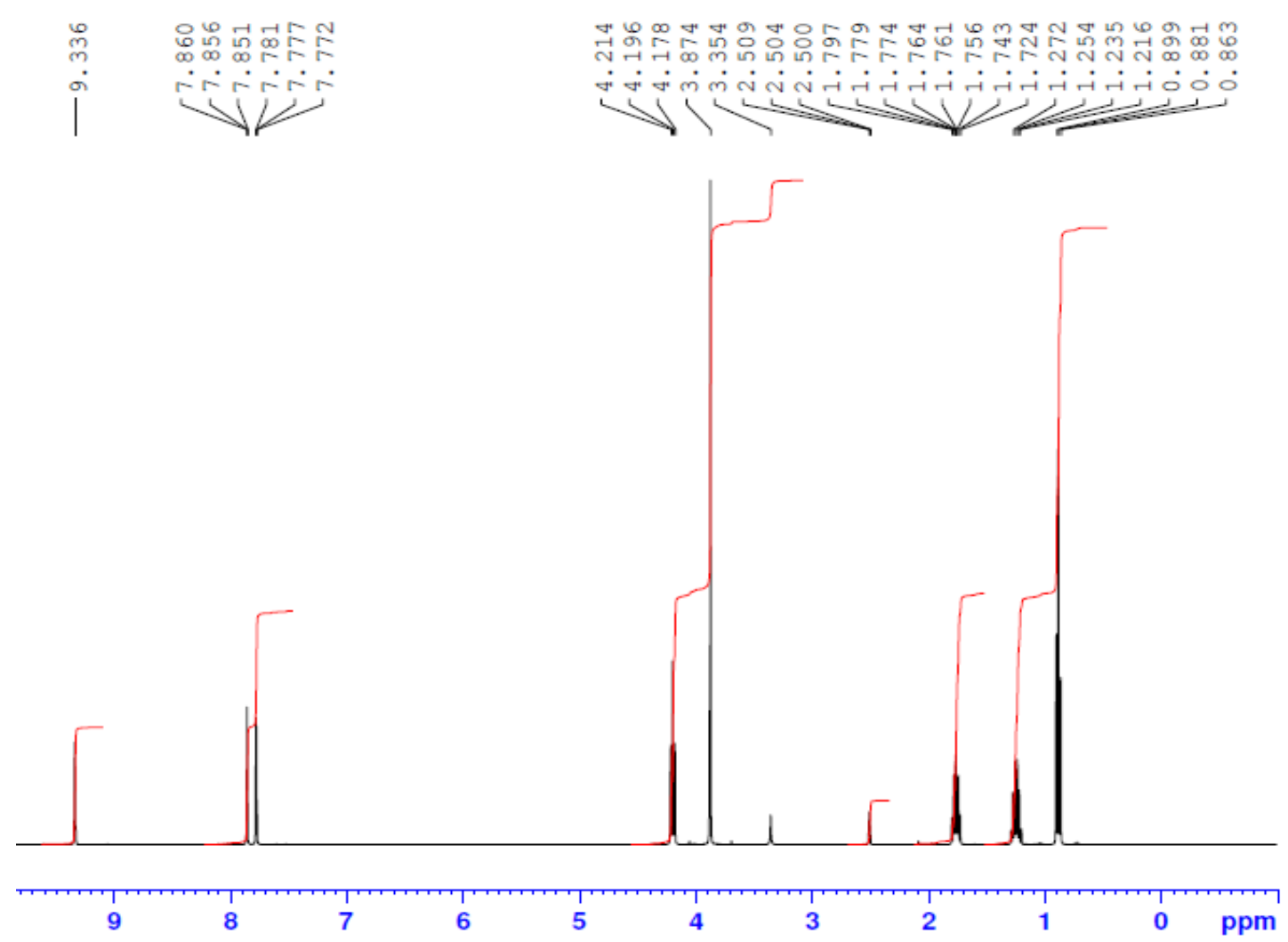

Figure S4 ${ }^{1} \mathrm{H}$ NMR spectrum of [bmim]Br in deuterated DMSO. 


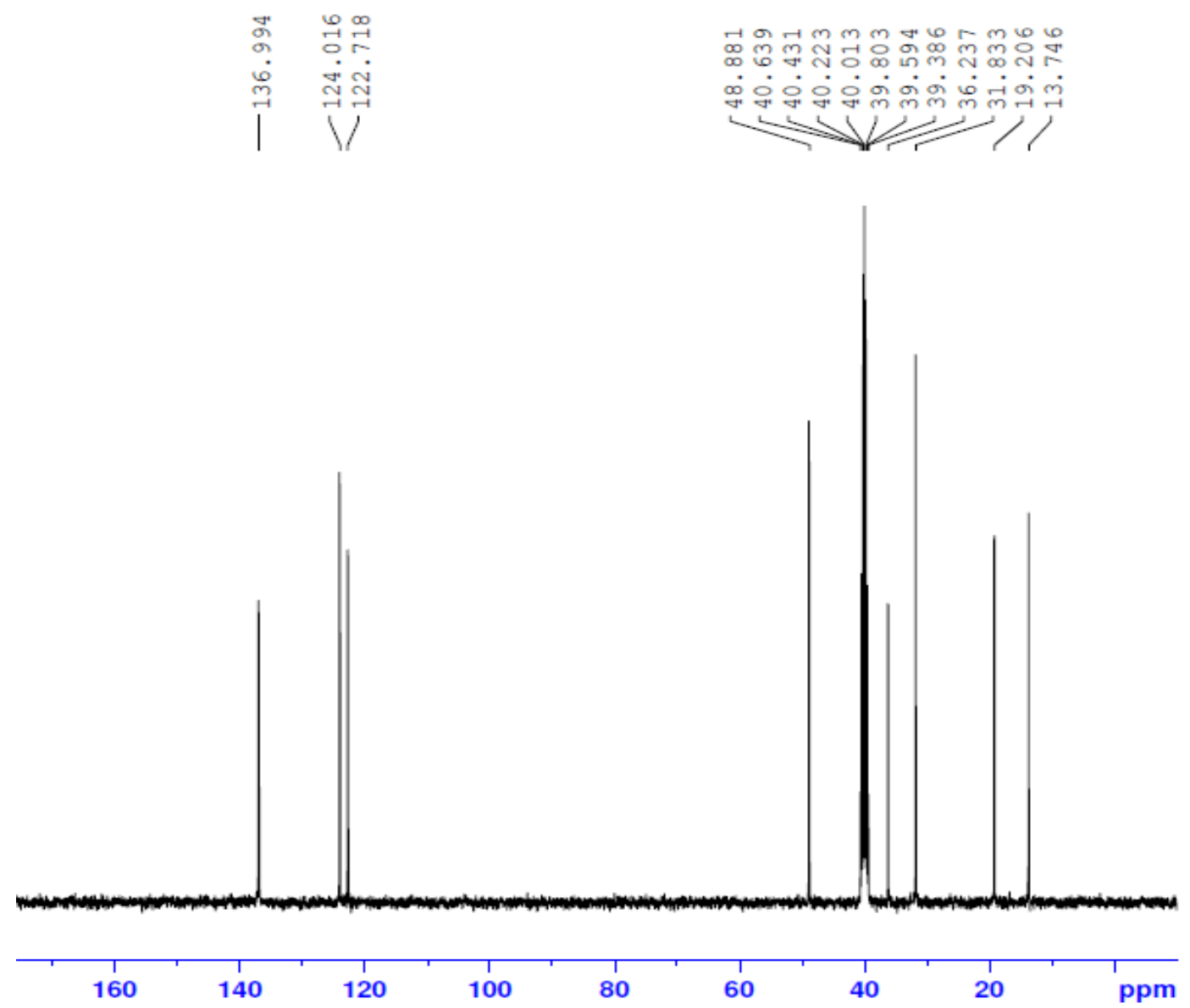

Figure S5 ${ }^{13} \mathrm{C}\left\{{ }^{1} \mathrm{H}\right\}$ NMR spectrum of [bmim] Br in deuterated DMSO.

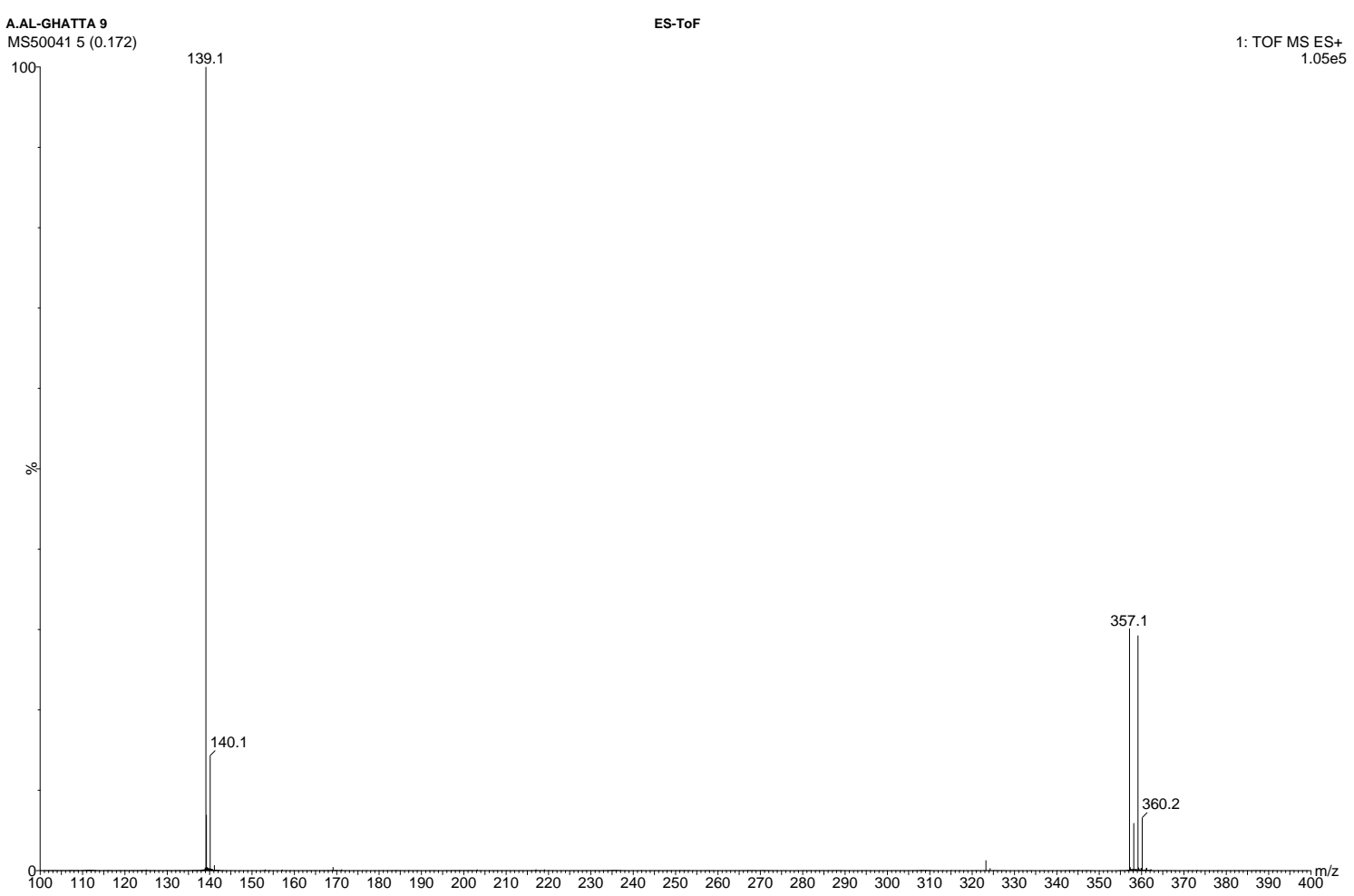

Figure S6 Mass spectrum of [bmim]Br. 


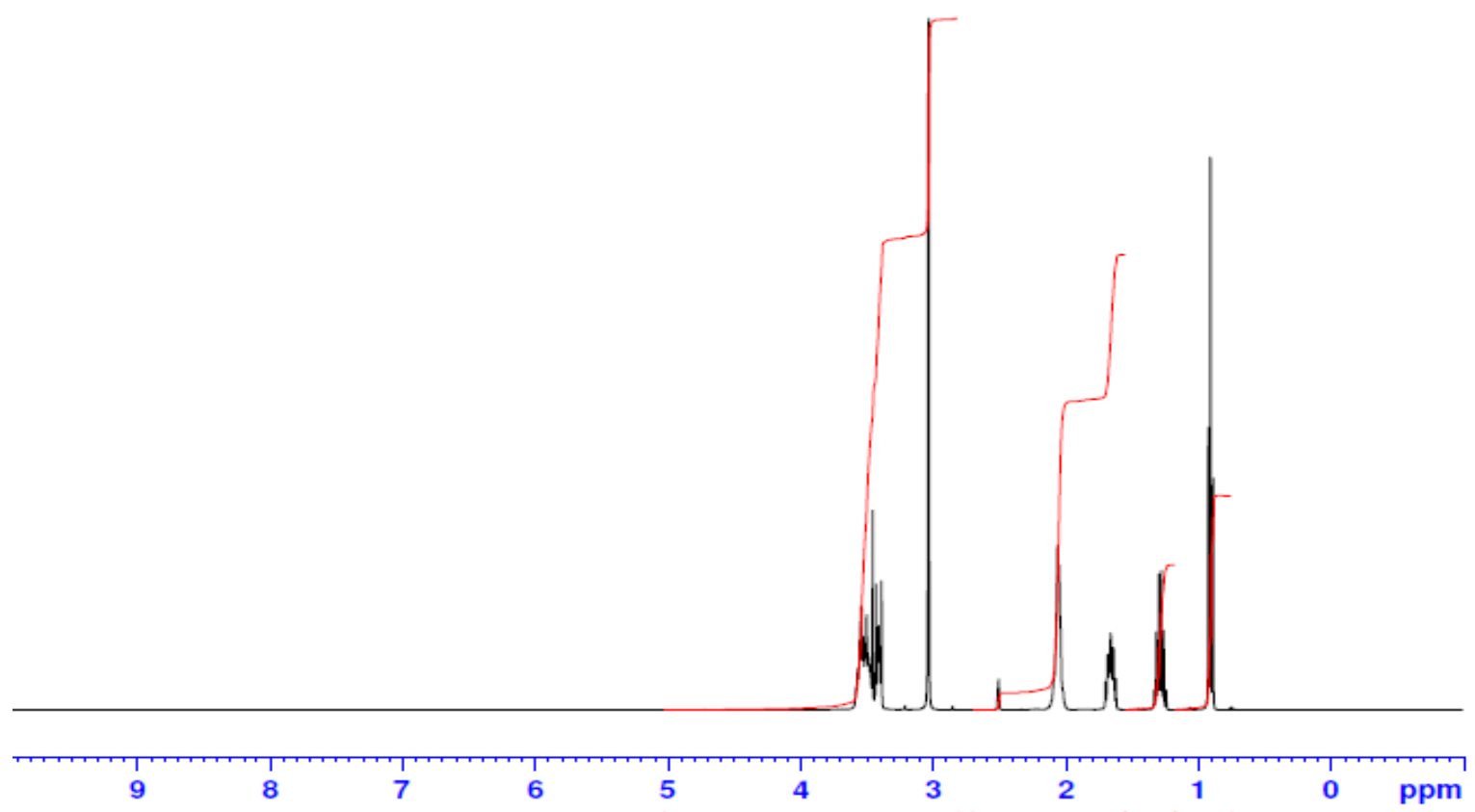

Figure $\mathbf{S} 7{ }^{1} \mathrm{H} N M R$ spectrum of [bmpyr]Cl in deuterated DMSO

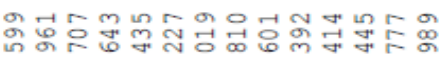

சं

प

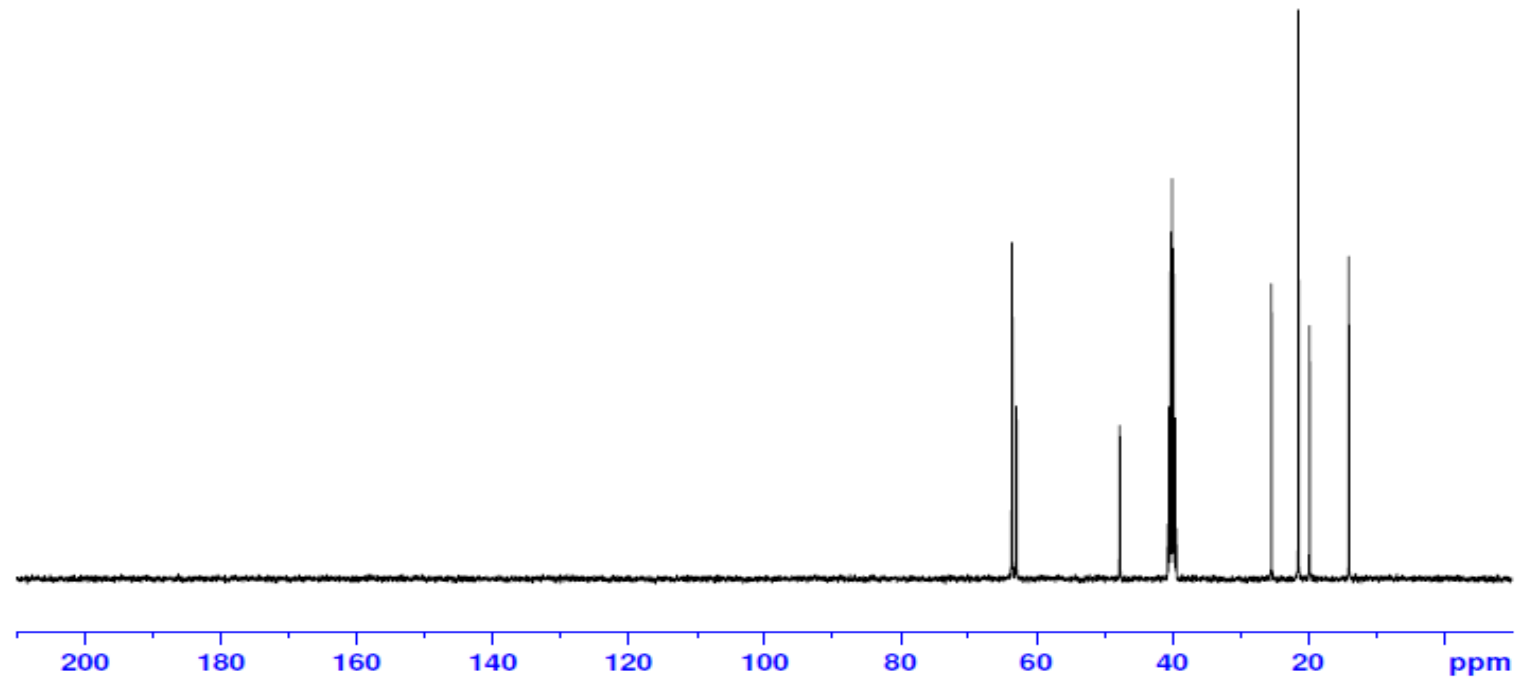

Figure S8 ${ }^{13} C\left\{{ }^{1} H\right\} N M R$ spectrum of [bmpyr]Cl in deuterated DMSO. 


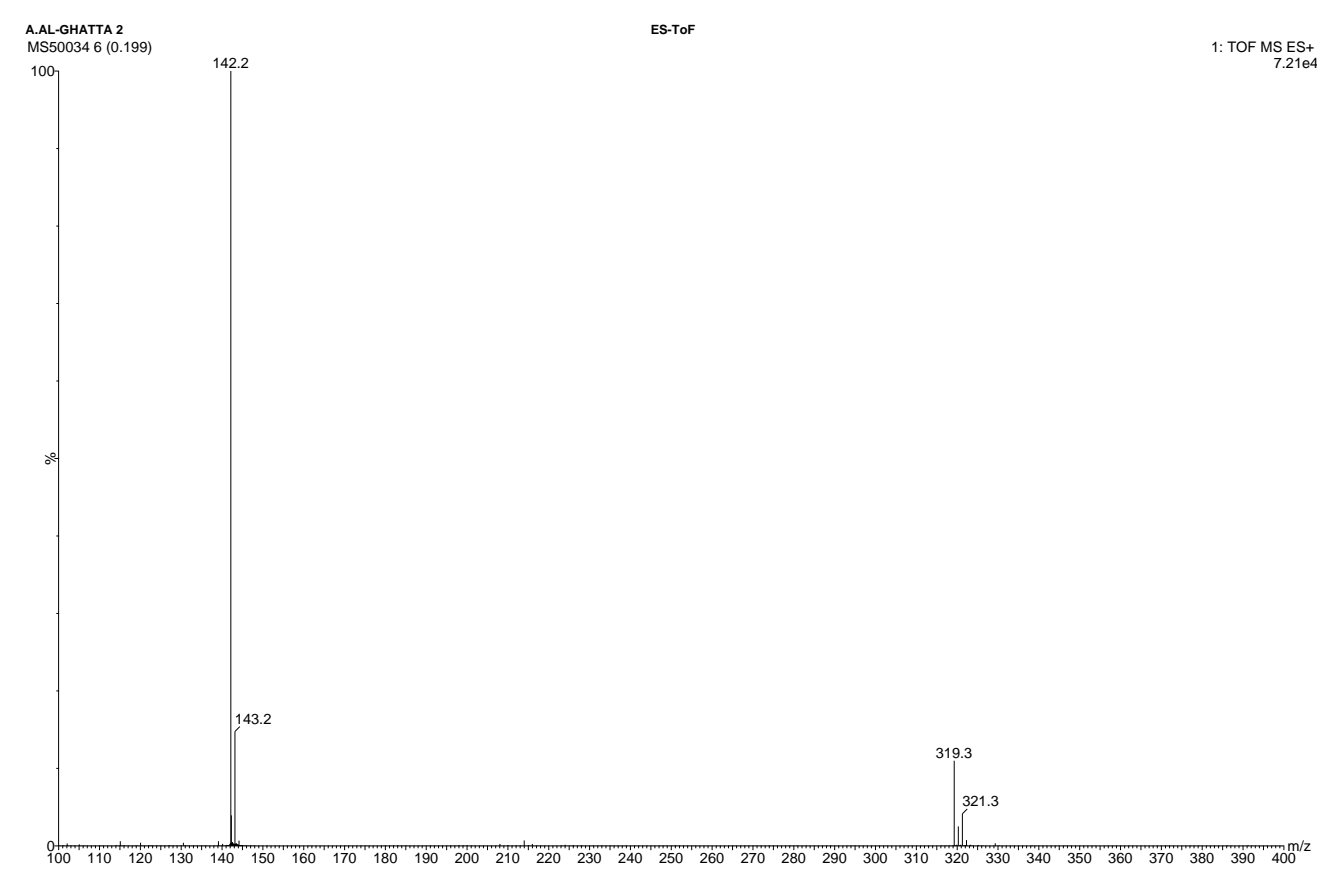

Figure S9 Mass spectrum of [bmpyr]Cl.

The characterisation data for $[\mathrm{bmim}] \mathrm{Cl},[\mathrm{bmim}] \mathrm{Br}$ and [bmpyr] $\mathrm{Cl}$ were in good agreement with those reported in the literature. ${ }^{\mathrm{S1}, \mathrm{S} 2}$

\section{S2.2 Synthesis of [bmim][OTf], [bmim][NTf 2 and [bmpyr][NTf 2$]$}

[bmim]Cl (30 g, $0.17 \mathrm{~mol})$ was dissolved in water $(100 \mathrm{~mL})$ and Li[OTf] $(30 \mathrm{~g}, 0.179 \mathrm{~mol}, 1.1$ eq) was dissolved in water $(50 \mathrm{~mL})$. The lithium triflate solution was added dropwise to the chloride solution under vigorous stirring and the reaction left overnight. The product was extracted from aqueous solution using a total of $800 \mathrm{~mL}$ dichloromethane. The organic extracts were washed repeatedly with water $(5 \mathrm{~mL})$ until the silver nitrate test revealed no halide ions (5-6 washes). The organic phase was dried with magnesium sulfate and then evaporated to dryness under reduced pressure. The ionic liquid, [bmim][OTf] was further dried under reduced pressure overnight at $60^{\circ} \mathrm{C}$ (final yield: $47 \mathrm{~g}, 96 \%$ ) and handled thereafter under an inert atmosphere. [bmim] $\left[\mathrm{NTf}_{2}\right]$ was synthesized by the same procedure using $\mathrm{Li}\left[\mathrm{NTf}_{2}\right]$ and [bmpyr]NTf $f_{2}$ was prepared by the same method using [bmpyr]Cl and $\mathrm{Li}_{[}\left[\mathrm{NTf}_{2}\right]$. The characterisation data for $[\mathrm{bmim}][\mathrm{OTf}],{ }^{, 4}[\mathrm{bmim}]\left[\mathrm{NTf}_{2}\right]^{\mathrm{s5}}$ and $[\mathrm{bmpyr}]\left[\mathrm{NTf}_{2}\right]^{\mathrm{S3}}$ were in good agreement with those reported in the literature.

[bmim][OTf]: ${ }^{1} \mathrm{H}$ NMR (DMSO- $\left.\mathrm{d}_{6}\right): \delta 9.08\left(\mathrm{~s}, 1 \mathrm{H}, \mathrm{N}_{2} \mathrm{CH}\right), 7.76-7.69-(\mathrm{m} \times 2,2 \times 1 \mathrm{H}, 2 \times \mathrm{N}-$ $\mathrm{CH}), 4.17\left(\mathrm{t}, 2 \mathrm{H}, \mathrm{N}-\mathrm{CH}_{2}-\mathrm{CH}_{2}, \mathrm{JHH}_{\mathrm{H}}=7.2 \mathrm{~Hz}\right), 3.85\left(\mathrm{~s}, 3 \mathrm{H}, \mathrm{N}-\mathrm{CH}_{3}\right), 1.77-1.26(\mathrm{~m} \times 2,2 \times 2 \mathrm{H}, \mathrm{N}-$ $\mathrm{CH}_{2}-\mathrm{C}_{2}-\mathrm{CH}_{2}-\mathrm{CH}_{3}$ ), 0.9 (t, 3H, $-\mathrm{CH}_{2}-\mathrm{CH}_{3}, J_{\mathrm{HH}}=7.4 \mathrm{~Hz}$ ) ppm. ${ }^{13} \mathrm{C}\left\{{ }^{1} \mathrm{H}\right\} \mathrm{NMR}$ (DMSO-d $\mathrm{d}_{6}$ ): $\delta 137.7$ $\left(\mathrm{N}_{2}-\mathrm{CH}\right), 123.6-122.4(2 \times \mathrm{N}-\mathrm{CH}), 119.2\left(\mathrm{CF}_{3}\right), 49.1\left(\mathrm{~N}-\mathrm{CH}_{2}-\mathrm{CH}_{2}-\mathrm{CH}_{2}-\mathrm{CH}_{3}\right), 35.8\left(\mathrm{NCH}_{3}\right), 32.0$ $\left(\mathrm{NCH}_{2} \mathrm{CH}_{2} \mathrm{CH}_{2} \mathrm{CH}_{3}\right), 19.0\left(\mathrm{NCH}_{2}-\mathrm{CH}_{2}-\mathrm{CH}_{2}-\mathrm{CH}_{3}\right), 13.8\left(\mathrm{~N}-\left(\mathrm{CH}_{2}\right)_{2}-\mathrm{CH}_{2}-\underline{\mathrm{C}} \mathrm{H}_{3}\right)$ ppm. MS (ES, +ve mode): $\mathrm{m} / \mathrm{z}$ (abundance) $=139(100)[\mathrm{bmim}]^{+}$. 
[bmim][NTf ${ }_{2}$ : ${ }^{1} \mathrm{H}$ NMR (DMSO-d $): \delta 9.33\left(\mathrm{~s}, 1 \mathrm{H}, \mathrm{N}_{2} \mathrm{CH}\right), 7.86,7.78(\mathrm{~m} \times 2,2 \times 1 \mathrm{H}, 2 \times \mathrm{N}-$ $\mathrm{CH}), 4.20\left(\mathrm{t}, 2 \mathrm{H}, \mathrm{N}-\mathrm{C}_{2}-\mathrm{CH}_{2}, J_{H H}=7.3 \mathrm{~Hz}\right), 3.87\left(\mathrm{~s}, 3 \mathrm{H}, \mathrm{N}-\mathrm{CH}_{3}\right), 1.76,1.25(\mathrm{~m} \times 2,2 \times 2 \mathrm{H}, \mathrm{N}-$ $\left.\mathrm{CH}_{2}-\underline{\mathrm{C}}_{2}-\mathrm{C}_{2}-\mathrm{CH}_{3}\right), 0.88$ (t, 3H, $\left.-\mathrm{CH}_{2}-\underline{\mathrm{C}}_{3}, J_{H H}=7.4 \mathrm{~Hz}\right) \mathrm{ppm} .{ }^{13} \mathrm{C}\left\{{ }^{1} \mathrm{H}\right\} \mathrm{NMR}\left(\mathrm{DMSO}-\mathrm{d}_{6}\right): \delta$ $136.7\left(\mathrm{~N}_{2}-\mathrm{CH}\right), 124.06,122.6(2 \times \mathrm{N}-\mathrm{CH}), 121.7\left(\mathrm{CF}_{3}\right) 48.9\left(\mathrm{~N}-\mathrm{CH}_{2}-\mathrm{CH}_{2}-\mathrm{CH}_{2}-\mathrm{CH}_{3}\right), 36.1$ $\left(\mathrm{NCH}_{3}\right), 31.7\left(\mathrm{NCH}_{2} \underline{\mathrm{C}} \mathrm{H}_{2} \mathrm{CH}_{2} \mathrm{CH}_{3}\right), 19.1\left(\mathrm{NCH}_{2}-\mathrm{CH}_{2}-\mathrm{CH}_{2}-\mathrm{CH}_{3}\right), 13.5\left(\mathrm{~N}-\left(\mathrm{CH}_{2}\right)_{2}-\mathrm{CH}_{2}-\underline{\mathrm{C}} \mathrm{H}_{3}\right) \mathrm{ppm}$. MS (ES, +ve mode): $\mathrm{m} / z$ (abundance) $=558(100)\left[(\mathrm{bmim})_{2} \mathrm{NTf}_{2}\right]^{+}, 139(25)[\mathrm{bmim}]^{+}$.

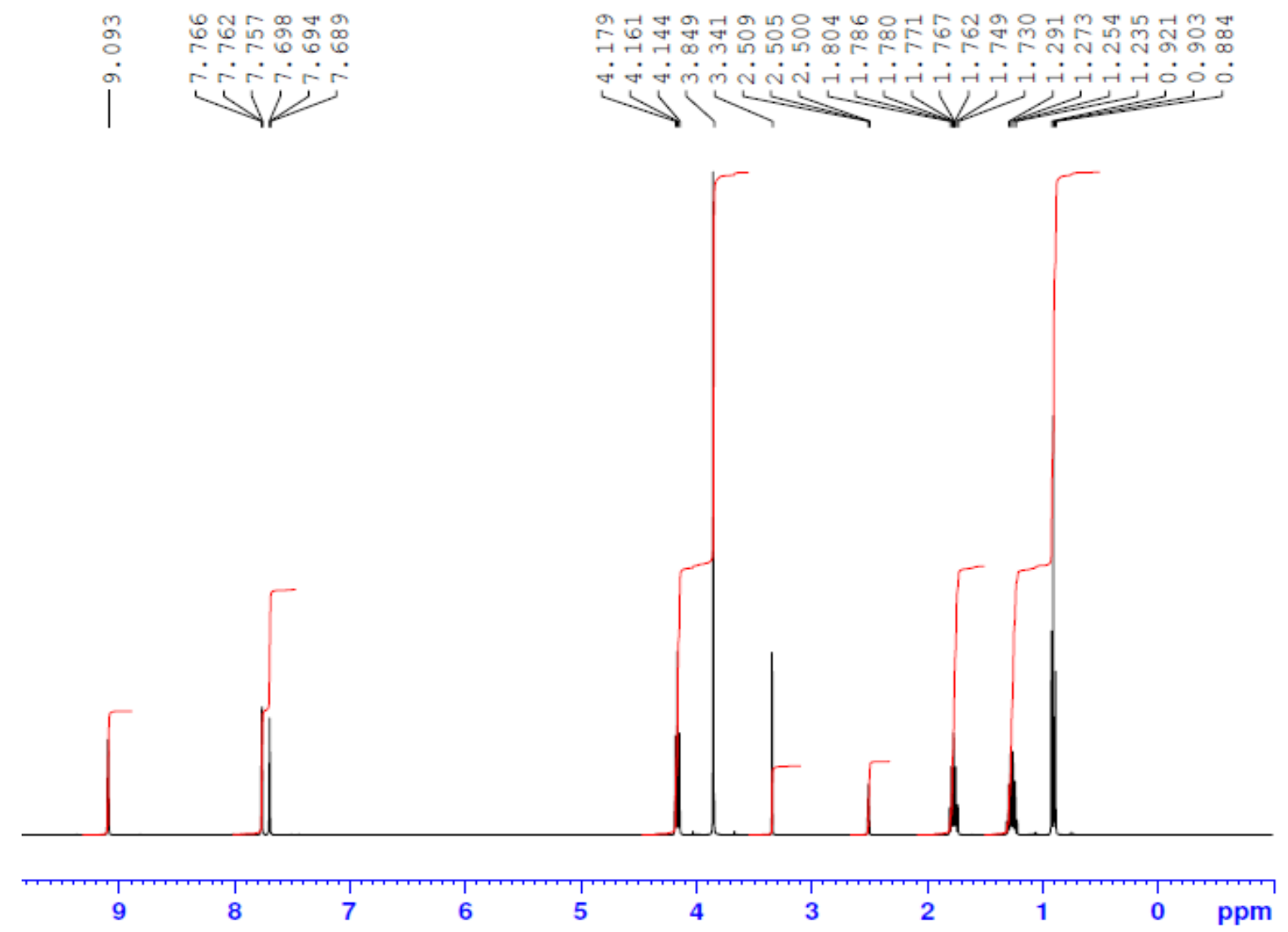

Figure $\mathrm{S} 10^{1} \mathrm{H}$ NMR spectrum of [bmim][OTf] in deuterated DMSO 


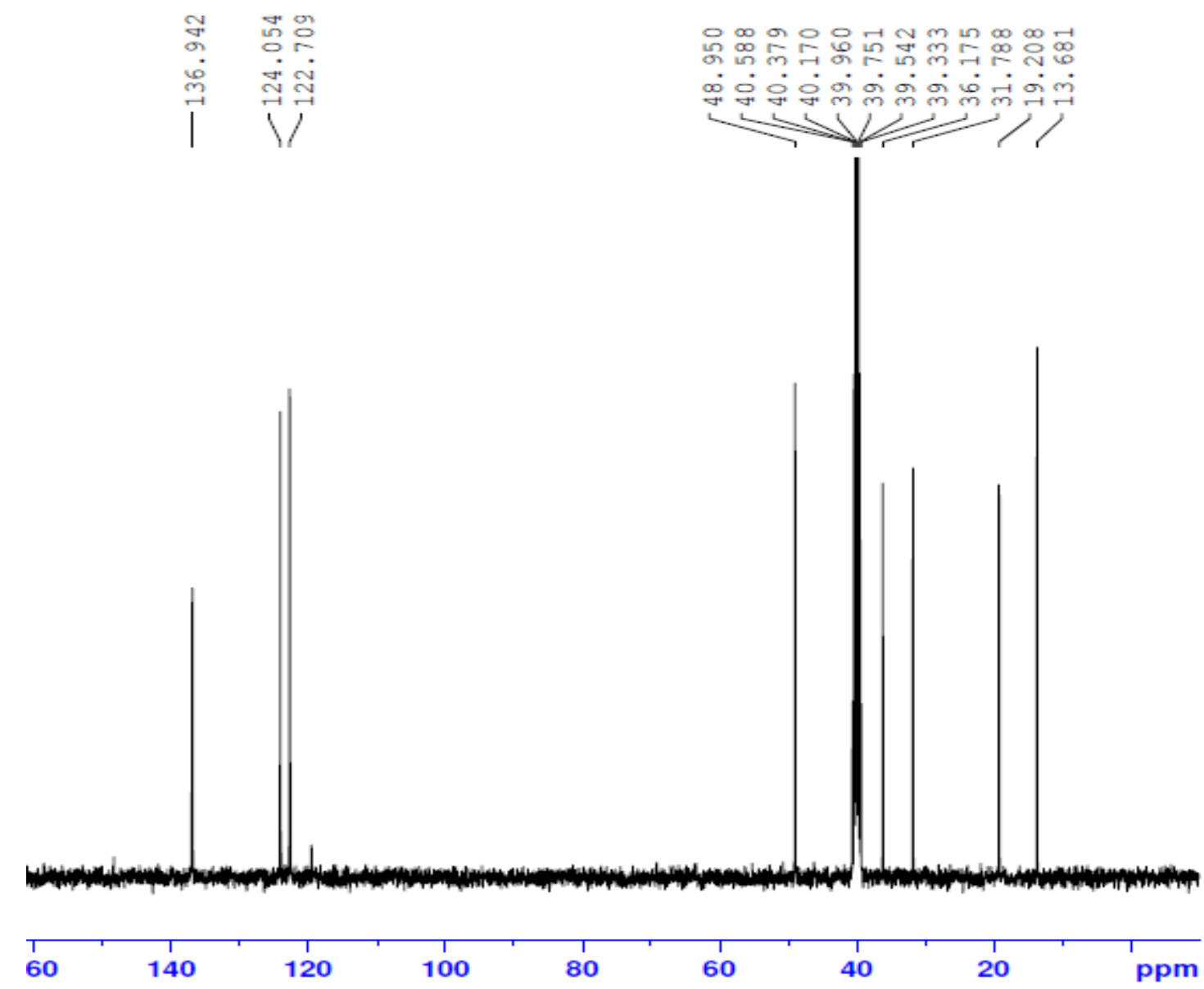

Figure $\mathrm{S} 11{ }^{13} \mathrm{C}\left\{{ }^{1} \mathrm{H}\right\}$ NMR spectrum of [bmim][OTf] in deuterated DMSO

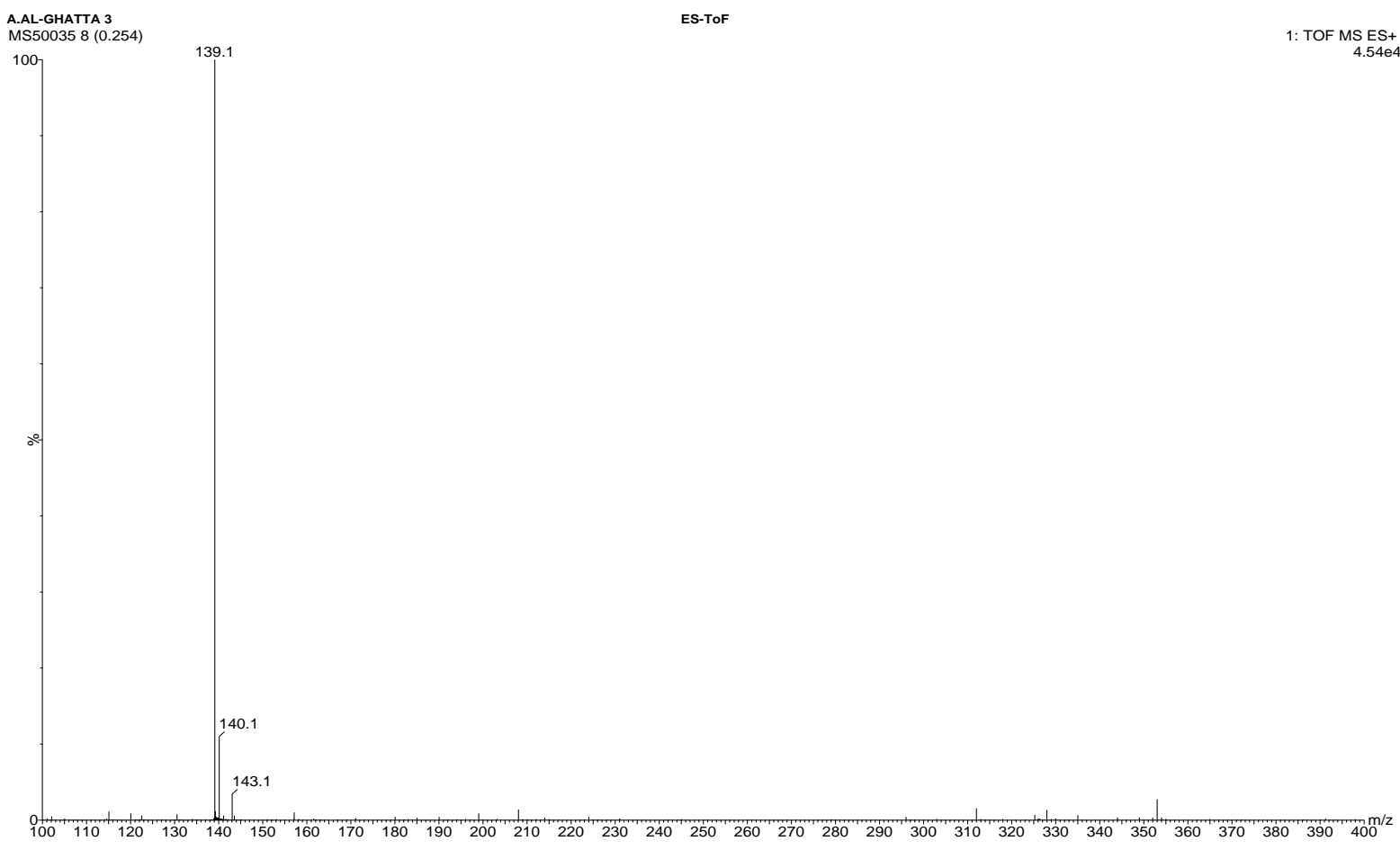

Figure S12 Mass spectrum of [bmim][OTf]. 


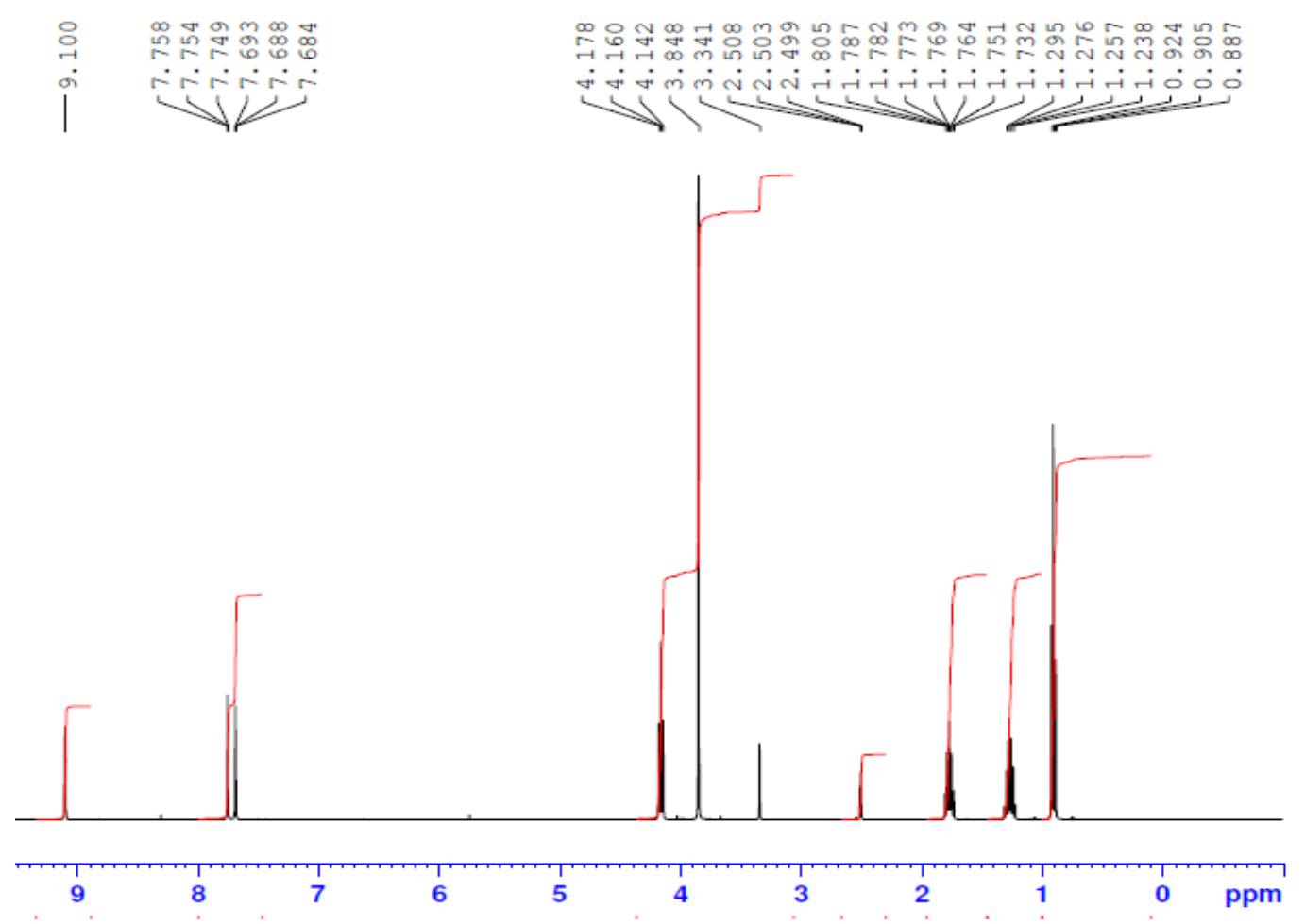

Figure $\mathrm{S} 13{ }^{1} \mathrm{H}$ NMR spectrum of $[\mathrm{bmim}]\left[\mathrm{NTf}_{2}\right]$ in deuterated DMSO.

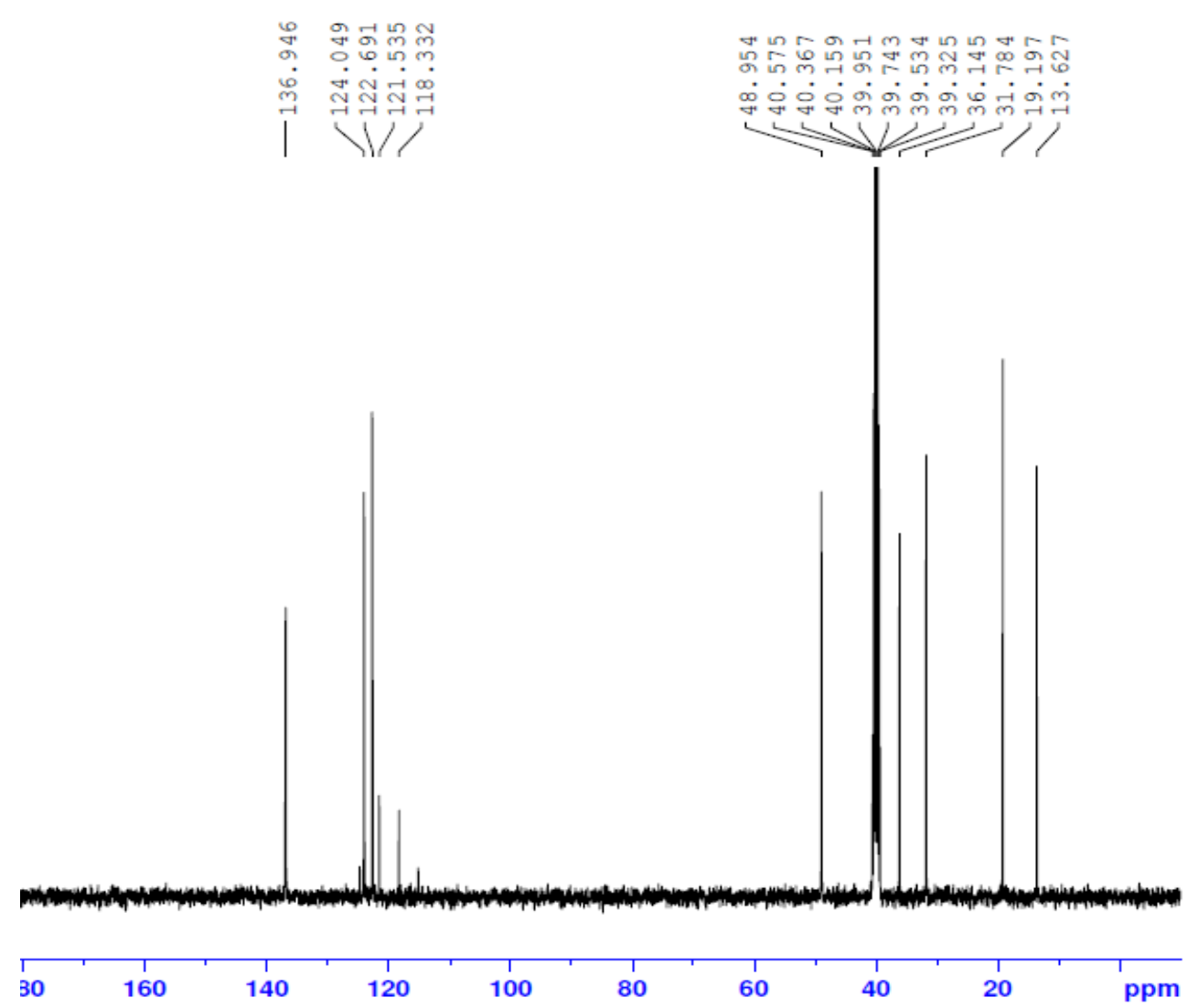

Figure $\mathbf{S} 14{ }^{13} \mathrm{C}\left\{{ }^{1} \mathrm{H}\right\}$ NMR spectrum of [bmim][NTf 2$]$ in deuterated DMSO. 


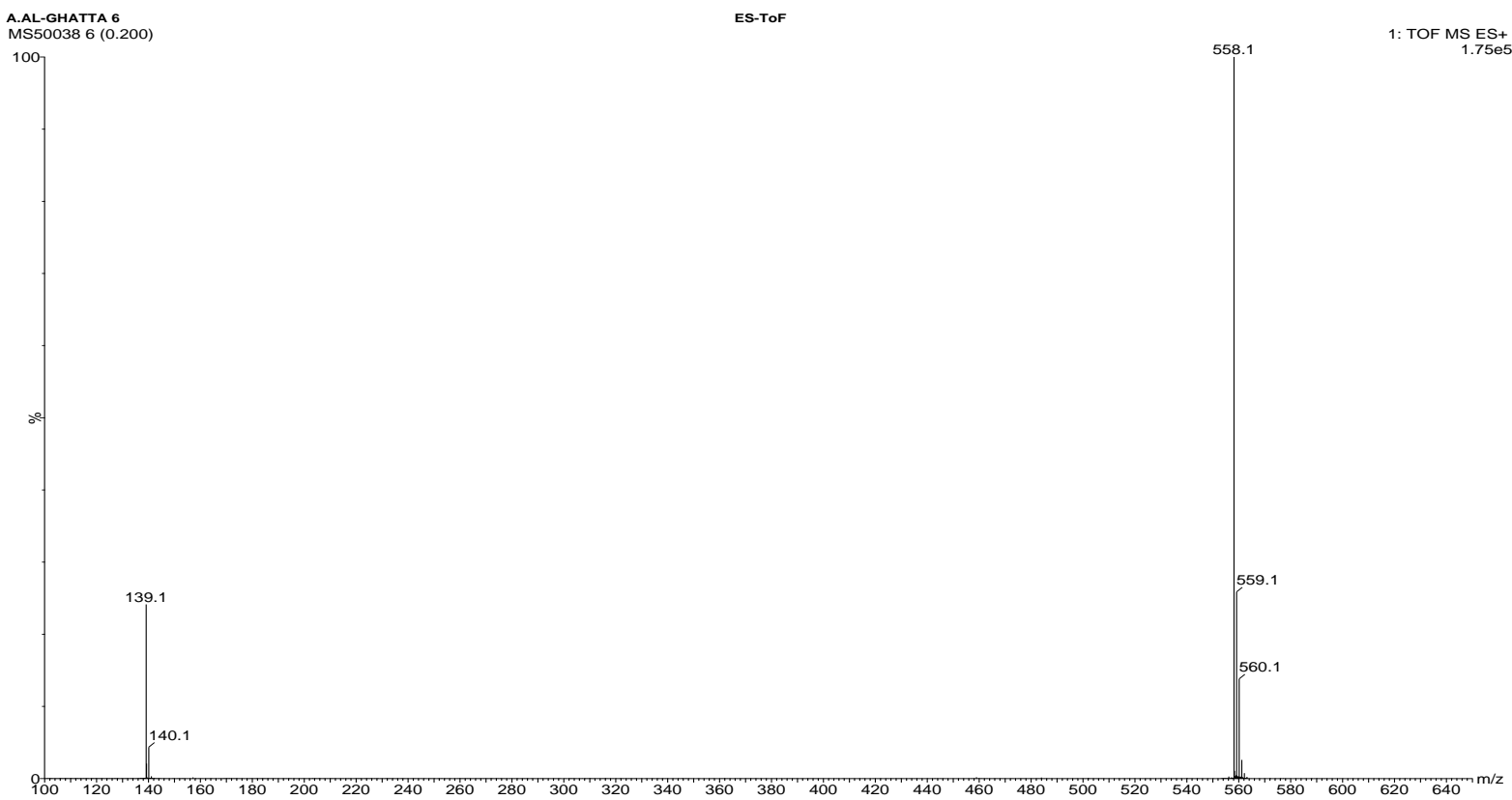

Figure S15 Mass spectrum of [bmim][ $\left.\mathrm{NTf}_{2}\right]$.

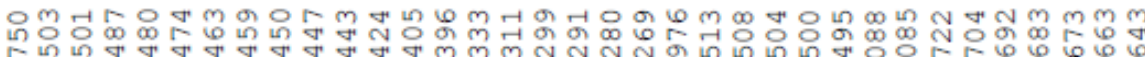

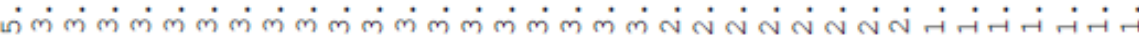

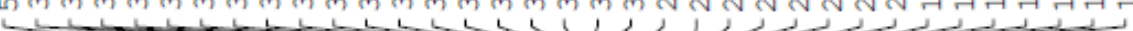

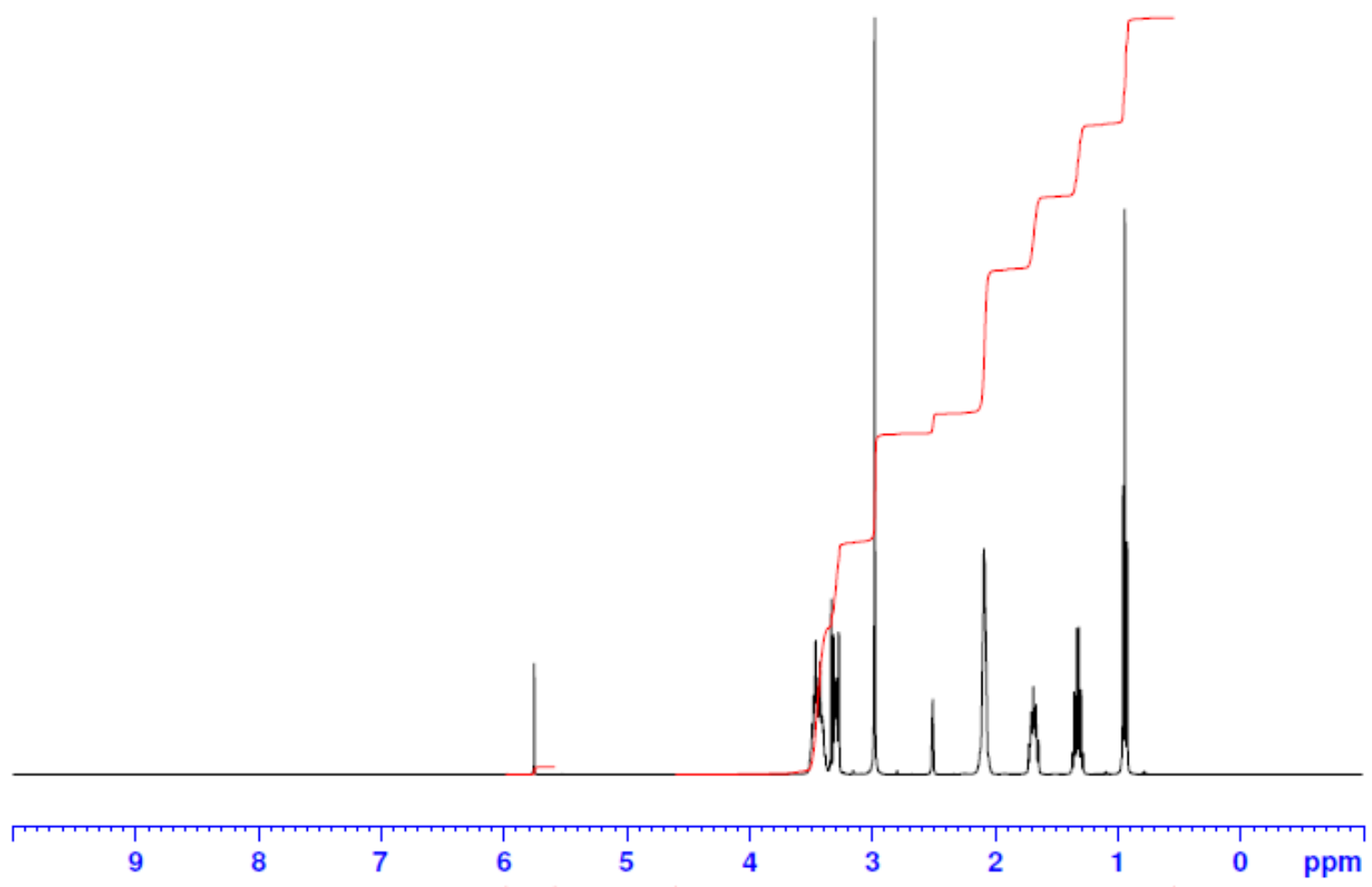

Figure $\mathrm{S} 16{ }^{1} \mathrm{H}$ NMR spectrum of $[\mathrm{bmpyr}]\left[\mathrm{NTf}_{2}\right]$ in deuterated DMSO. 


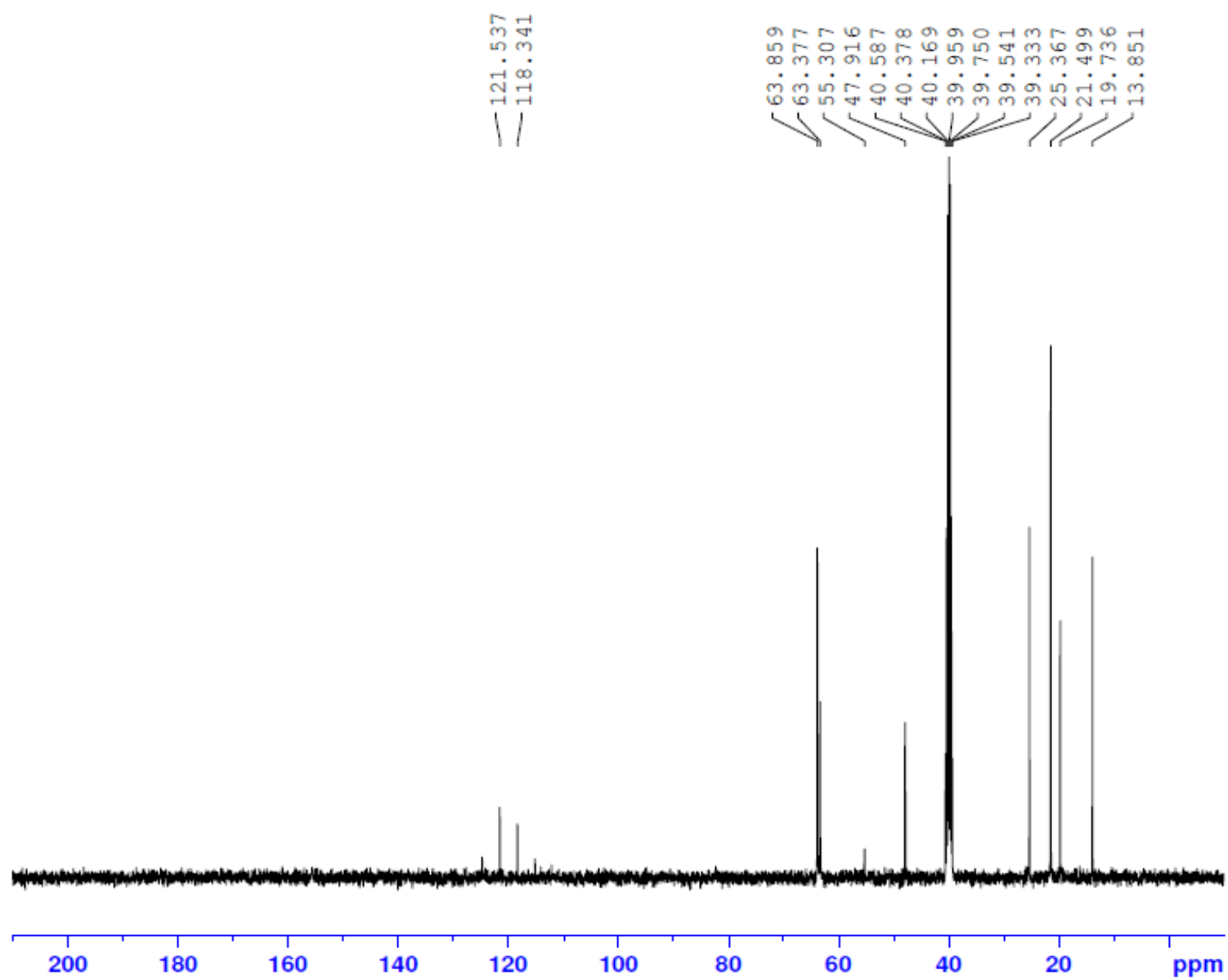

Figure $17{ }^{13} \mathrm{C}\left\{{ }^{1} \mathrm{H}\right\}$ NMR spectrum of [bmpyr] $\left[\mathrm{NTf}_{2}\right]$ in deuterated DMSO.

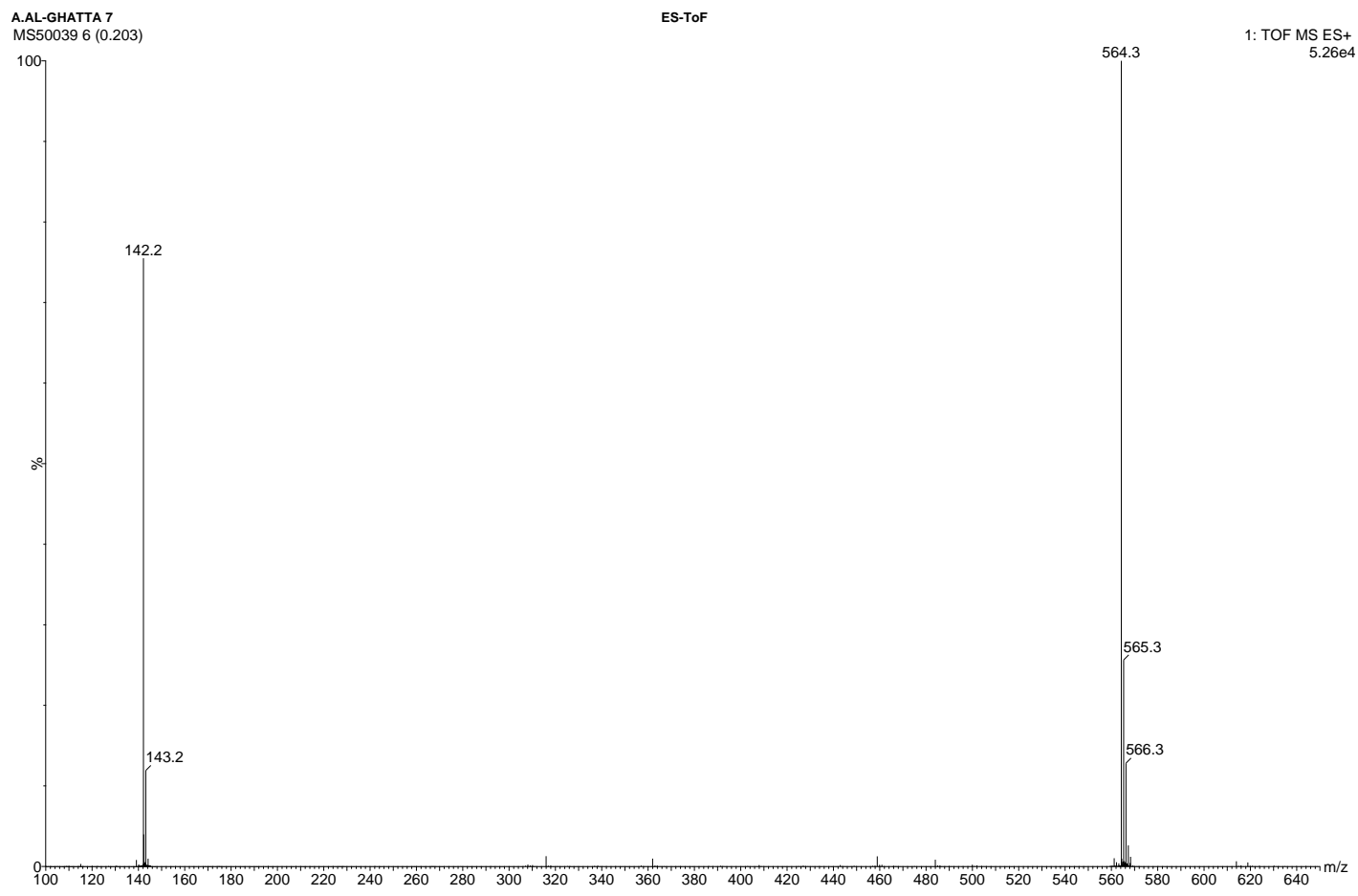

Figure S18 Mass spectrum of [bmpyr][NTf 2$]$ 


\section{S3 Aspen Simulation}

Simulations were performed with Aspen Plus 8.8V and the ILUAM package using the thermodynamic model COSMOSAC. ${ }^{\mathrm{S} 6}$ The initial feed conditions chosen were at $100{ }^{\circ} \mathrm{C}$ and 1.2 bar.

Table S1 Summary of conditions for drying [bmim] Cl from initial 52\% water content

\begin{tabular}{cccc}
\hline Stage & Pressure $($ bar $)$ & Temperature $\left({ }^{\circ} \mathrm{C}\right)$ & Duty $(\mathrm{KJ} / \mathrm{Kg})$ \\
\hline 1 & 0.8 & 93.5 & 0.409 \\
2 & 0.5 & 86 & 0.385 \\
3 & 0.2 & 76 & 0.333 \\
4 & 0.01 & 7 & 0.263
\end{tabular}

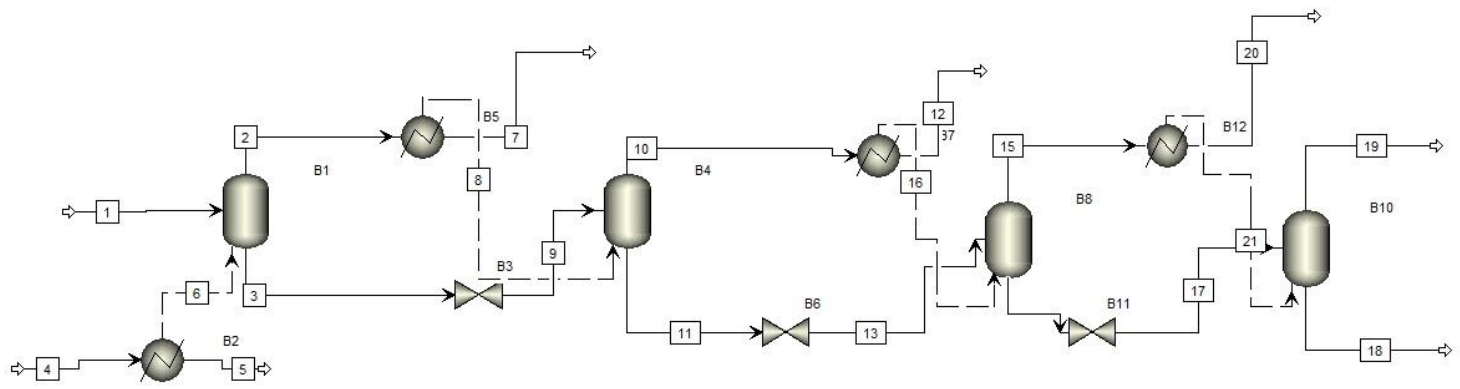

Figure S19 Aspen flowsheet for 4-stage evaporators.

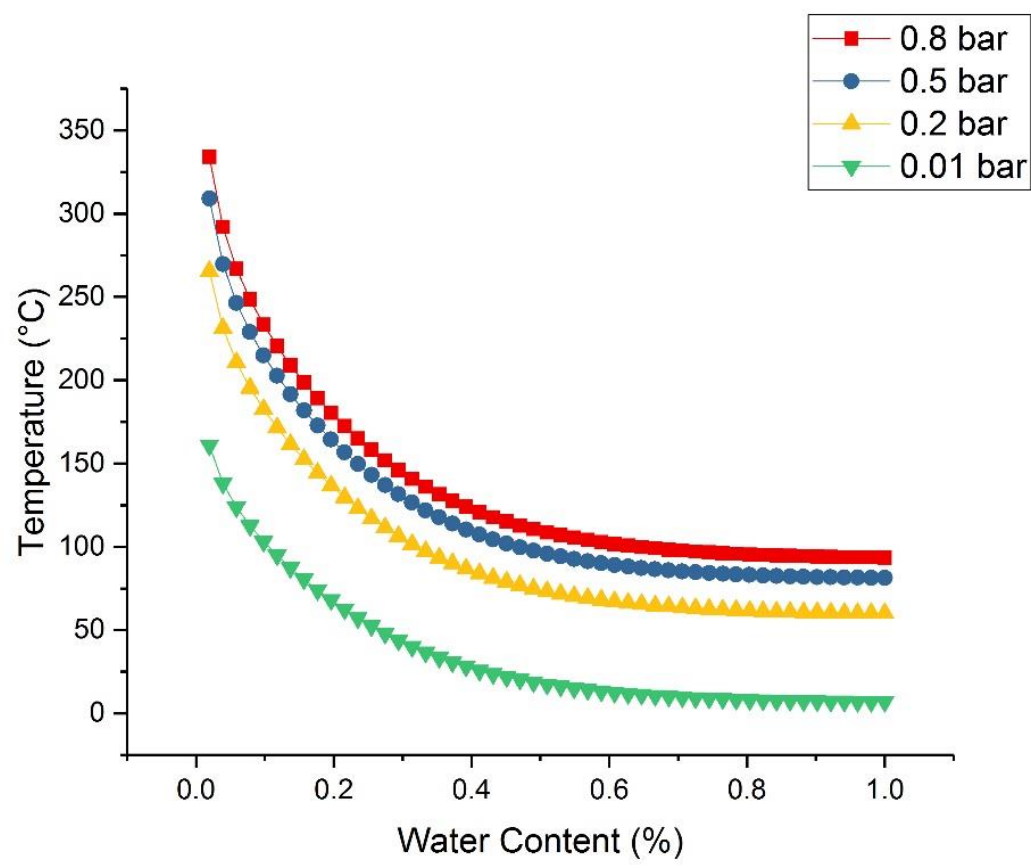

Figure S20 Equilibrium diagram between [bmim]Cl/water estimated by the COSMOSAC model. 


\section{S4 Sublimation of DFF}

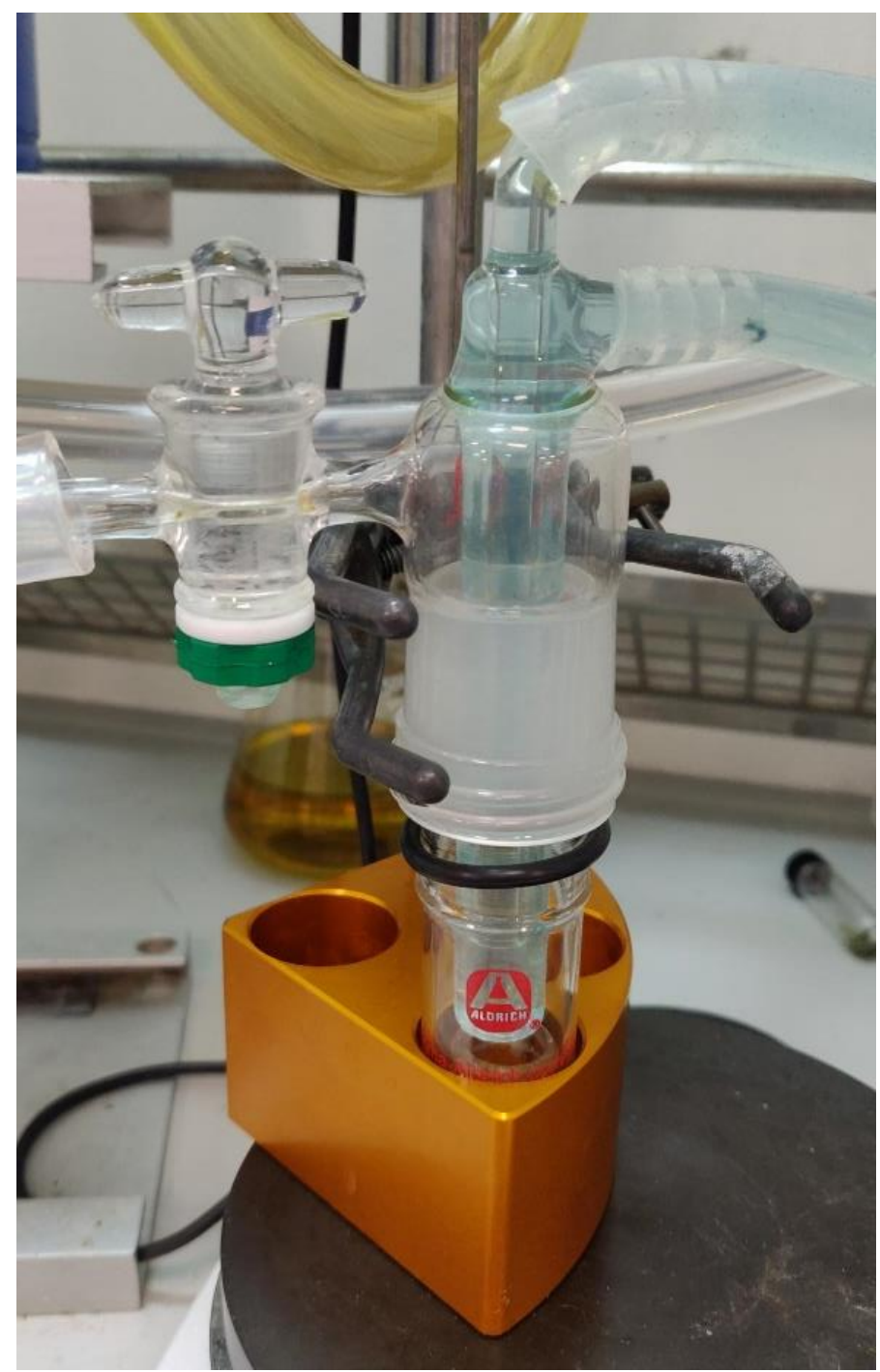

Figure S21 Cold finger apparatus for sublimation of DFF.
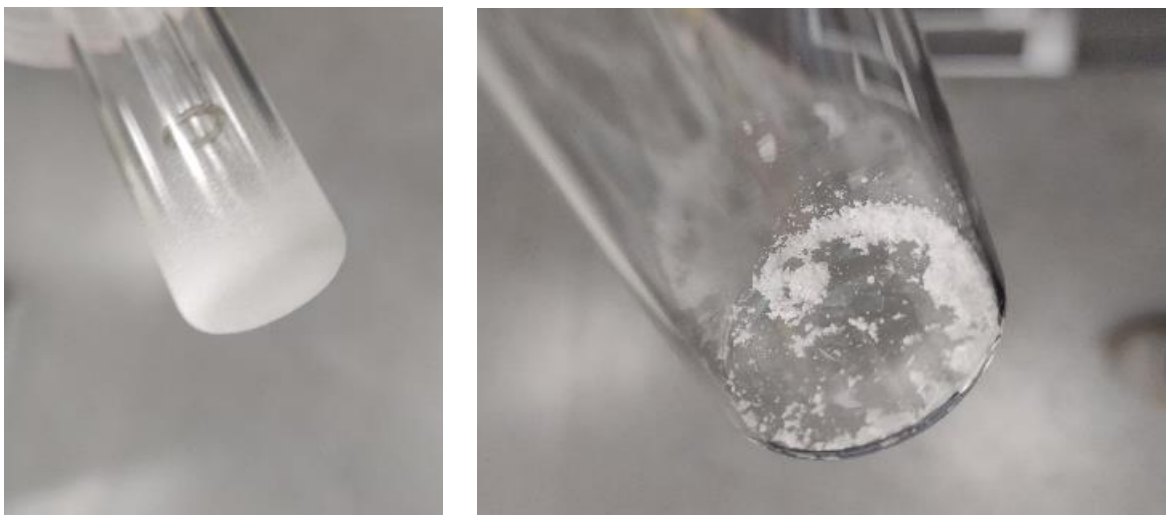

Figure S22 Sublimed DFF from the IL on the condenser tip (left) and collected DFF (right). 


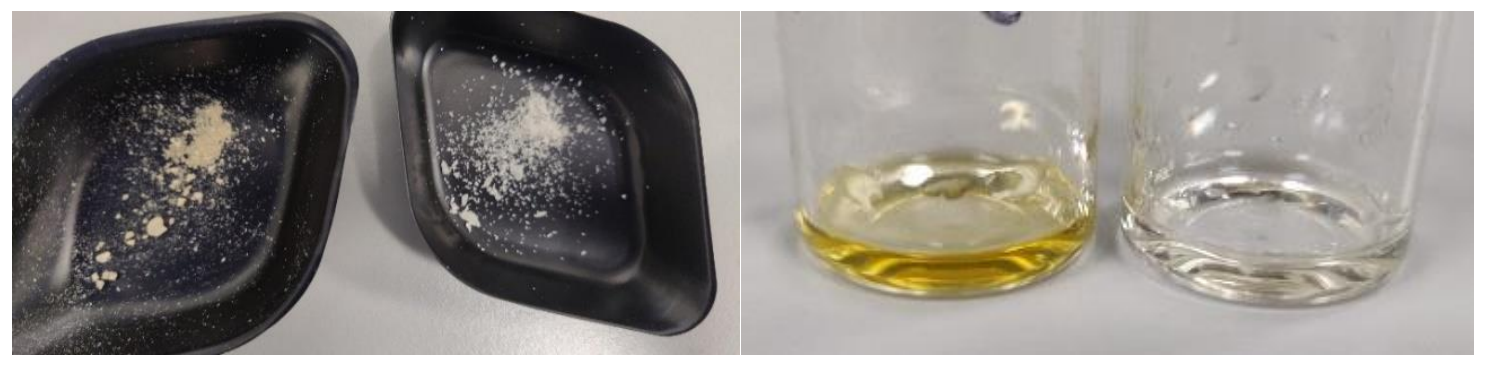

Figure S23 Comparison between the commercial DFF and the sublimed material (left) and comparison of the bulk IL after first sublimation and second sublimation (right).

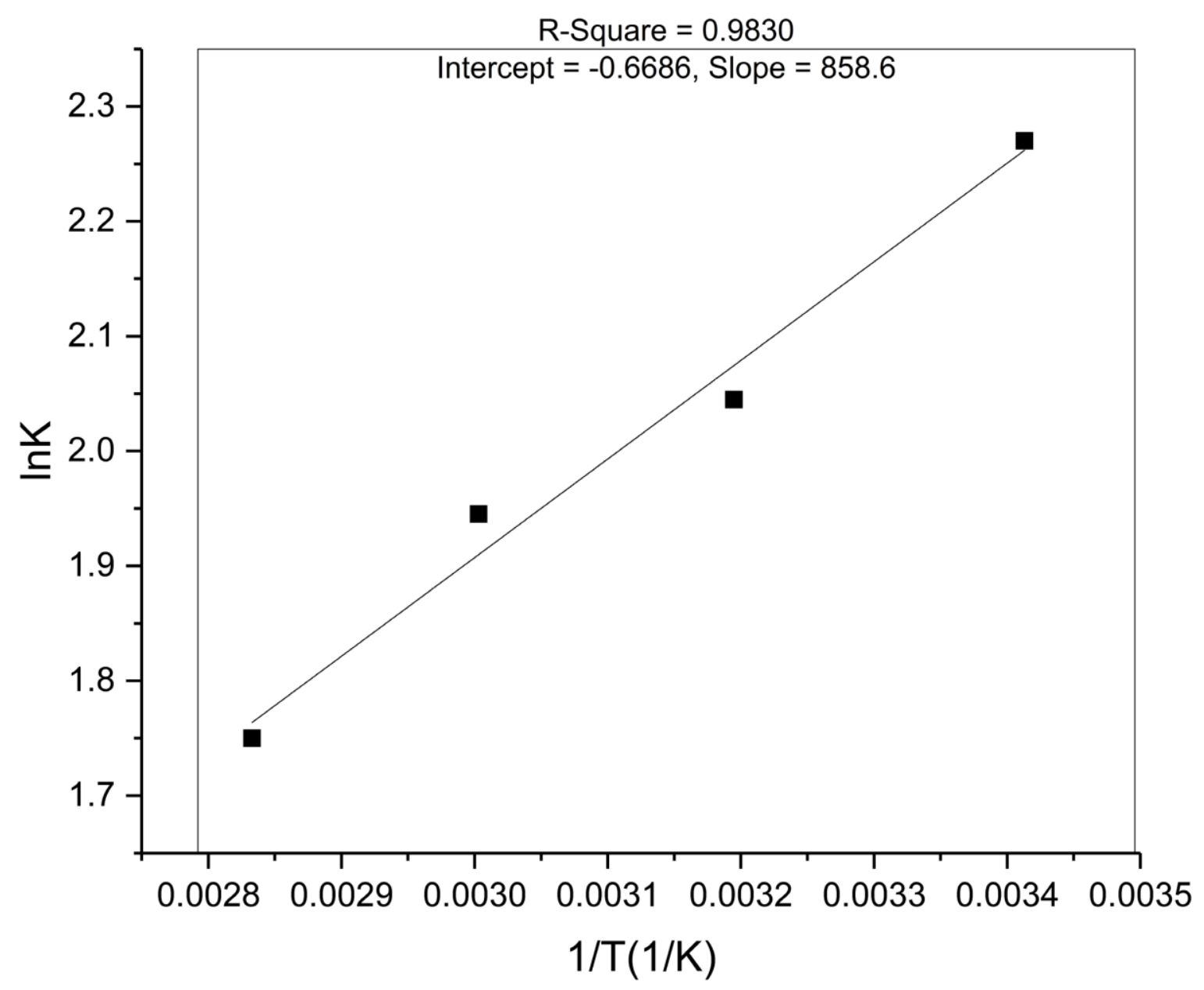

Figure S24 Fitting of the partition coefficient with temperature using the Arrhenius equation for $\mathrm{HMF}$ in $\left[\mathrm{N}(\mathrm{Me}) \mathrm{Oct}_{3}\right] \mathrm{Cl}$ and water. 


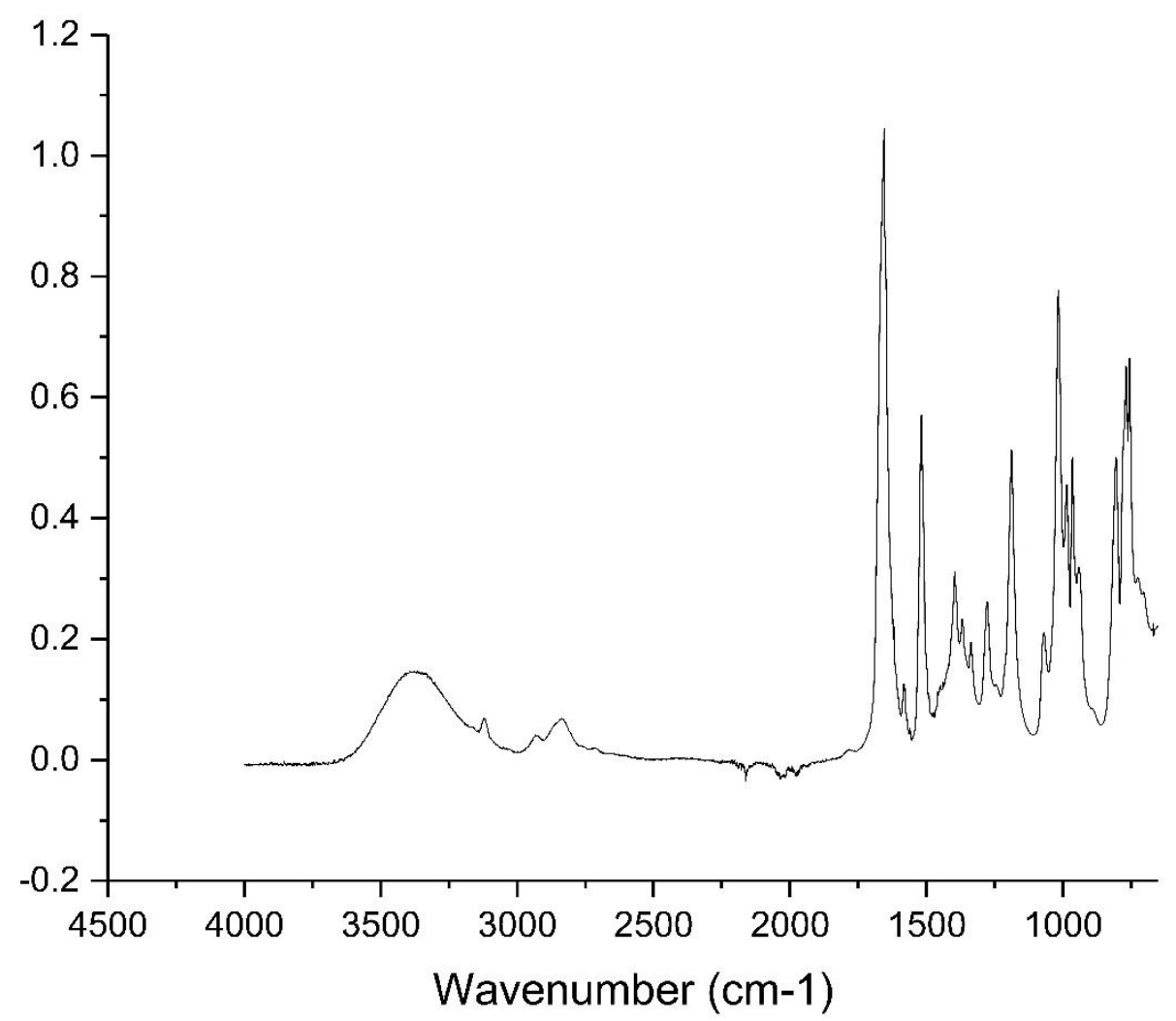

Figure S25 Infrared spectrum of pure HMF (transmission mode).
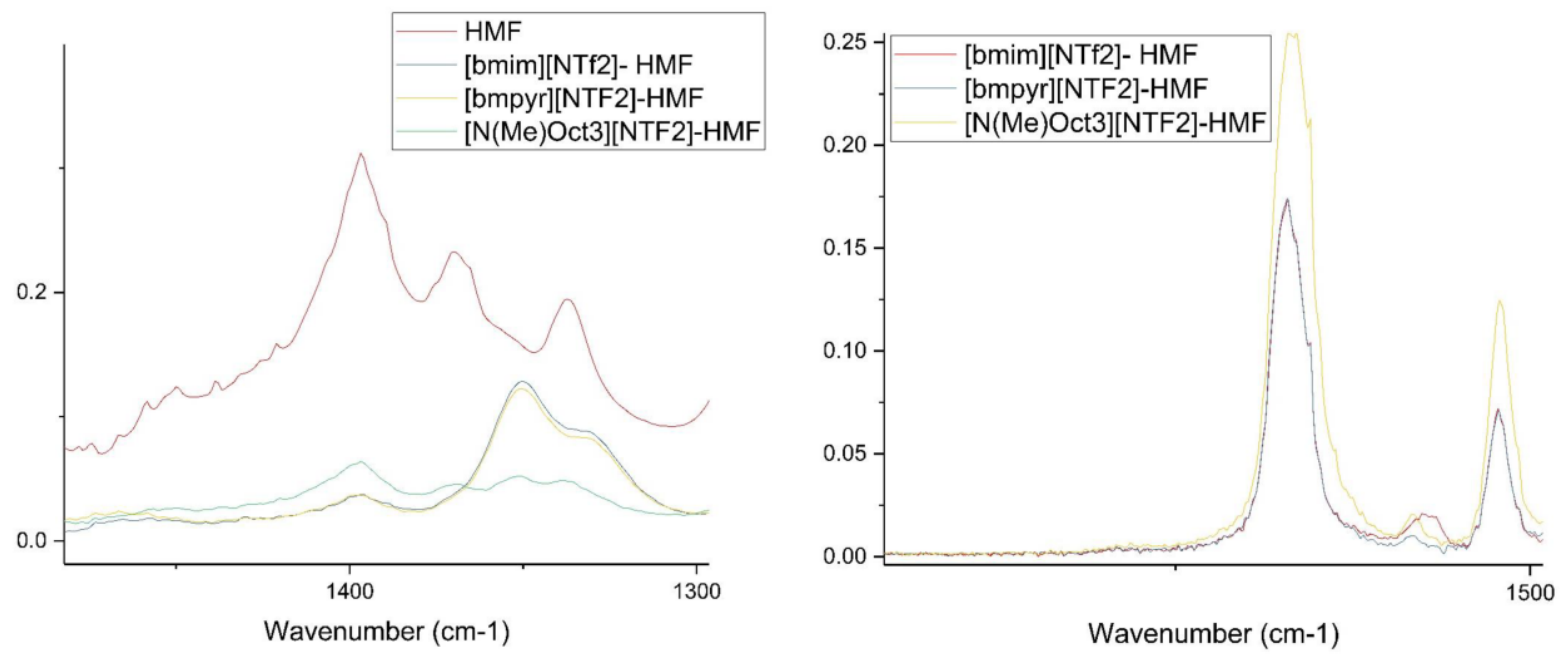

Figure S26 Cation effect on the infrared data (transmission mode) associated with the furan ring (left) and carbonyl group (right) of HMF. 


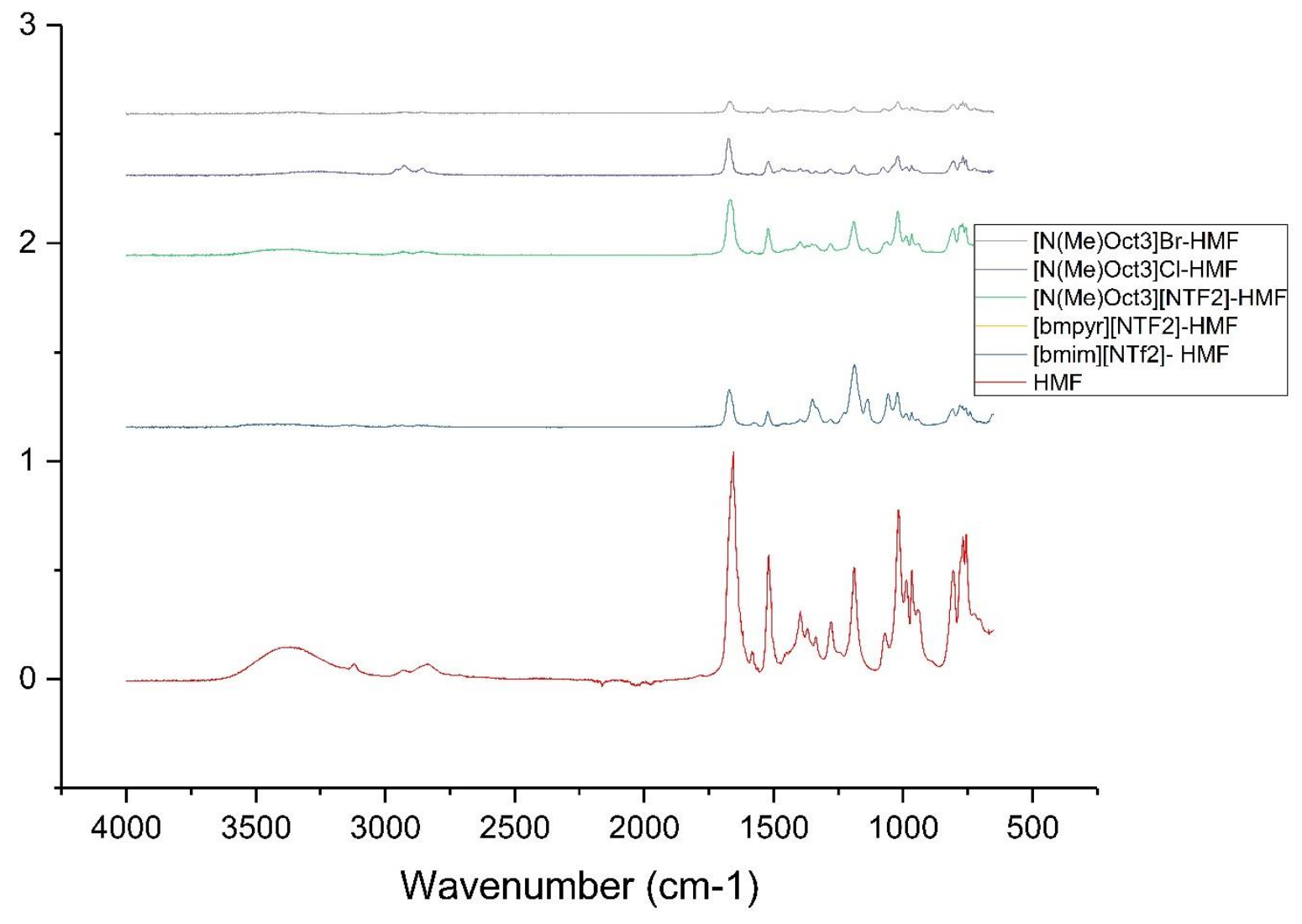

Figure S27 Infrared spectra of HMF in various ionic liquids (transmission mode). 


\section{S5 Numerical data}

Table S2 Recovery of DFF in $6 \mathrm{~h}$ at different temperatures from [bmim][NTf 2 under vacuum.

\begin{tabular}{cc}
\hline Temperature $\left({ }^{\circ} \mathrm{C}\right)$ & Recovery $(\%)$ \\
\hline 40 & $7.1 \pm 2.0$ \\
60 & $95.2 \pm 1.2$ \\
80 & $96.8 \pm 0.3$ \\
100 & $97.0 \pm 0.4$
\end{tabular}

Table S3 Solubility data for FFCA and FDCA in [bmim]Cl/water mixtures.

\begin{tabular}{ccc}
\hline Water content $(\%)$ & FFCA $(\mathrm{mg} / \mathrm{g})$ & FDCA $(\mathrm{mg} / \mathrm{g})$ \\
\hline 20 & $238.4 \pm 2.4$ & $113.5 \pm 1.1$ \\
30 & $169.5 \pm 4.5$ & $70.1 \pm 2.9$ \\
40 & $123.6 \pm 8.63$ & $36.5 \pm 2.1$ \\
50 & $72.2 \pm 0.6$ & $18.0 \pm 0.1$ \\
60 & $39.9 \pm 0.5$ & $10.7 \pm 0.6$ \\
80 & $15.5 \pm 0.5$ & $6.0 \pm 0.01$ \\
100 & $9.1 \pm 1.5$ & $3.0 \pm 0.0$ \\
\hline
\end{tabular}

Table S4 Solubility data for FFCA and FDCA in [bmim]Br/water mixtures.

\begin{tabular}{ccc}
\hline Water content $(\%)$ & FFCA $(\mathrm{mg} / \mathrm{g})$ & FDCA $(\mathrm{mg} / \mathrm{g})$ \\
\hline 20 & $91.3 \pm 1.5$ & $34.4 \pm 0.4$ \\
30 & $75.2 \pm 0.4$ & $22.7 \pm 0.8$ \\
40 & $58.6 \pm 0.5$ & $15.8 \pm 0.22$ \\
50 & $43.2 \pm 1.1$ & $10.7 \pm 0.7$ \\
60 & $29.6 \pm 0.1$ & $5.9 \pm 0.0$ \\
80 & $17 \pm 0.5$ & $4.0 \pm 0.3$ \\
100 & $9.1 \pm 1.5$ & $3.0 \pm 0.0$ \\
\hline
\end{tabular}

Table S5 Solubility data for FFCA and FDCA in [bmim][OTf]/water mixtures.

\begin{tabular}{ccc}
\hline Water content $(\%)$ & FFCA $(\mathrm{mg} / \mathrm{g})$ & FDCA $(\mathrm{mg} / \mathrm{g})$ \\
\hline 0 & $12.6 \pm 0.4$ & $3.4 \pm 0.1$ \\
20 & $24.2 \pm 0.3$ & $10.1 \pm 0.1$ \\
30 & $15.0 \pm 0.3$ & $5.7 \pm 0.0$ \\
40 & $15.5 \pm 0.3$ & $5.9 \pm 0.2$ \\
50 & $16.15 \pm 1.5$ & $5.6 \pm 0.1$ \\
\hline
\end{tabular}


Table S6 Partition coefficient of $\left[\mathrm{N}(\mathrm{Me}) \mathrm{Oct}_{3}\right] \mathrm{Cl} /$ water mixtures at different temperatures.

\begin{tabular}{cc}
\hline $\mathrm{T}\left({ }^{\circ} \mathrm{C}\right)$ & $\mathrm{K}$ \\
\hline 20 & $9.7 \pm 0.0$ \\
40 & $7.7 \pm 0.0$ \\
60 & $7.0 \pm 0.1$ \\
80 & $6.4 \pm 0.9$ \\
\hline
\end{tabular}

Table S7 Anion effect on the partition coefficient of $\left[\mathrm{N}(\mathrm{Me}) \mathrm{Oct}_{3}\right]^{+}$salts with water at $20^{\circ} \mathrm{C}$.

\begin{tabular}{cc}
\hline Anion & $\mathrm{K}$ \\
\hline $\mathrm{Cl}^{-}$ & $9.7 \pm 0$ \\
$\mathrm{Br}^{-}$ & $10.4 \pm 0.1$ \\
{$\left[\mathrm{NTf}_{2}\right]^{-}$} & $2.3 \pm 0.1$ \\
\hline
\end{tabular}

Table S8 Cation effect on the partition coefficient for $\left[\mathrm{NTf}_{2}\right]^{-}$salts at $20^{\circ} \mathrm{C}$.

\begin{tabular}{cc}
\hline Cation & $\mathrm{K}$ \\
\hline$\left[\mathrm{Bmim}^{+}\right.$ & $3.0 \pm 0.0$ \\
${\text { Bmpyr }]^{+}}^{+}$ & $2.7 \pm 0.0$ \\
{$\left[\mathrm{~N}(\mathrm{Me}) \mathrm{Oct}_{3}\right]^{+}$} & $2.3 \pm 0.1$ \\
\hline
\end{tabular}

Table S9 Final water concentration (M) for different $\mathrm{HMF}$ loadings in $\left[\mathrm{N}(\mathrm{Me}) \mathrm{Oct}_{3}\right] \mathrm{Cl}$ at various phase ratios.

\begin{tabular}{ccccc}
\hline HMF loading & $\mathrm{R}=2$ & $\mathrm{R}=4$ & $\mathrm{R}=6$ & $\mathrm{R}=8$ \\
\hline 100 & 0.27 & 0.26 & 0.24 & 0.22 \\
60 & 0.16 & 0.15 & 0.14 & 0.14 \\
40 & 0.11 & 0.10 & 0.10 & 0.09 \\
20 & 0.05 & 0.05 & 0.05 & 0.04 \\
10 & 0.03 & 0.026 & 0.024 & 0.02 \\
6.7 & 0.02 & 0.017 & 0.016 & 0.015 \\
3.3 & 0.01 & 0.008 & 0.008 & 0.007 \\
\hline
\end{tabular}




\section{S6 References}

S1. Eminov, S.; Brandt, A.; Wilton-Ely, J. D. E. T.; Hallett, J. P. The highly selective and near-quantitative conversion of glucose to 5-hydroxymethylfurfural using ionic liquids, PLoS One 2016, 11, 1-15, DOI 10.1371/journal.pone.0163835

S2. Correia, I.; Welton, T. An Old Reaction in New Media: Kinetic Study of a Platinum(II) Substitution Reaction in Ionic Liquids. Dalton Trans. 2009, 4115-4121, DOI 10.1039/B822152A.

S3. Jamehbozorg, B.; Sadeghi, R. Vapor Pressure Osmometry Studies of Aqueous lonic Liquid-Carbohydrate Systems, J. Chem. Eng. Data 2018, 63, 331-340, DOI 10.1021/acs.jced.7b00719.

S3. Wells, T. P.; Hallett, J. P.; Williams, C. K.; Welton, T. Esterification in ionic liquids: the influence of solvent basicity. J. Org. Chem. 2008, 73, 5585-5588, DOI 10.1021/j08005864.

S4. Tsurumaki, A.; Ohno, H. Dissolution of oligo(tetrafluoroethylene) and preparation of poly(tetrafluoroethylene)-based composites by using fluorinated ionic liquids, Chem. Commun. 2018, 4, 409-412, DOI 10.1039/C7CC08449H.

S5. Kuzmina, O.; Hassan, N. H.; Patel, L.; Ashworth, C.; Bakis, E.; White, A. J. P.; Hunt, P. A.; Welton, T. The impact of ionic liquids on the coordination of anions with solvatochromic copper complexes, Dalton Trans. 2017, 46, 12185-12200, DOI 10.1039/C7DT02372C.

S6. $\quad$ Ferro, V. R.; Moya, C.; Moreno, D.; Santiago, R.; De Riva, J.; Pedrosa, G.; Larriba, M.; Diaz, I.; Palomar, J. Enterprise lonic Liquids Database (ILUAM) for Use in Aspen ONE Programs Suite with COSMO-Based Property Methods. Ind. Eng. Chem. Res. 2018, 57, 980-989, DOI 10.1021/acs.iecr.7b04031. 